\title{
Effective Einstein cosmological spaces for non-minimal modified gravity
}

\author{
Emilio Elizalde $^{1}$ • Sergiu I. Vacaru ${ }^{2,3}$
}

Received: 8 December 2014 / Accepted: 28 April 2015 / Published online: 13 May 2015

(C) Springer Science+Business Media New York 2015

\begin{abstract}
Certain off-diagonal vacuum and nonvacuum configurations in Einstein gravity can mimic physical effects of modified gravitational theories of $f\left(R, T, R_{\mu \nu} T^{\mu \nu}\right)$ type. We prove this statement by constructing exact and approximate solutions which encode certain models of covariant Hořava type gravity with dynamical Lorentz symmetry breaking. Off-diagonal generalizations of de Sitter and nonholonomic $\Lambda \mathrm{CDM}$ universes are constructed which are generated through nonlinear gravitational polarization of fundamental physical constants and which model interactions with non-constant exotic fluids and effective matter. The problem of possible matter instability for such off-diagonal deformations in (modified) gravity theories is discussed.
\end{abstract}

Keywords Cosmological solutions of modified gravities - Accelerated expansion of the universe - Reconstruction procedures $\cdot f(R)$-gravities and generalizations . $\Lambda \mathrm{CDM}$ cosmology

Sergiu I. Vacaru

sergiu.vacaru@uaic.ro

Emilio Elizalde

elizalde@ieec.uab.es

1 Instituto de Ciencias del Espacio, Consejo Superior de Investigaciones Científicas, ICE-CSIC and IEEC, Campus UAB, Facultat de Ciències, Torre C5-Parell-2a planta, 08193 Bellaterra, Barcelona, Spain

2 Theory Division, CERN, 1211 Geneva 23, Switzerland

3 Rector's Department, Alexandru Ioan Cuza University, Alexandru Lapuşneanu Street, nr. 14, UAIC - Corpus R, Office 323, 700057 Iasi, Romania 


\section{Introduction}

There are various extensions of general relativity, GR, theory. Some of the most popular are $f(R), f(R, T)$, and $f(\mathbf{R}, \mathbf{T}, F)$ — which we will here generically call $f$ modified theories, or modified gravity theories, MGTs. For such modifications, the standard Lagrangian for GR, namely as $\mathcal{L}=R$, on a pseudo-Riemannian manifold, $V$-where $R$ is the Ricci scalar curvature for the Levi-Civita connection, $\nabla$-is modified by the addition of a functional, $f(R, \ldots)$, of the Ricci scalar only, in the first case, of $R$ and the torsion tensor, for a "boldface" symbol $\mathbf{T}=\left\{T_{\beta \gamma}^{\alpha}\right\}$, the energy-momentum tensor for matter, $T_{\beta \gamma}$, and/or its trace $T=T_{\alpha}^{\alpha}$ (in the second), and of a generalized Ricci scalar $\mathbf{R}$, and a Finsler generating function, $F$, in the third case (such values may be defined on the tangent bundle $T V$ ), etc. Classes of MGTs of these kinds can be successfully constructed. Corresponding reconstruction procedures, able to mimic the $\Lambda \mathrm{CDM}$ model including the dark energy epochs and the transitions between the different main stages of the universe evolution can be elaborated, see reviews of results in Refs. [1-10].

Several MGTs are actually related with different forms of the so-called covariant Hořava gravity associated with a dynamical breaking of Lorentz invariance [11-14], and with further developments, as well, including generic off-diagonal solutions, Lagrange-Hamilton-Finsler like generalizations, A-brane models, and gauge like gravity [15-19]. Theories of this kind can be constructed in a power-counting renormalizable form or as nonholonomic brane configurations which correspond to power-law versions of actions of type $f\left(R, T, R_{\mu \nu} T^{\mu \nu}\right)$ [20-29]. In general, such models are with commutative and/or noncommutative parameters, and off-diagonal metrics for wapred/trapped solutions, Lorentz violations, nonlinear dispersion relations and locally anisotropic re-scaling, and effective polarizations of constants [30-36].

Gravitational and matter field equations in GR and various MGTs usually consist of very sophisticated systems of nonlinear partial differential equations (PDEs) and request advanced numeric, analytic and geometric techniques for constructing exact and approximate solutions. After a series of assumptions of "high symmetry" of the relevant interactions (for spherical, cylindrical or torus ansätze, with a possible additional Lie group interior symmetry), such systems of nonlinear PDEs are usually transformed into much more simplified systems of nonlinear ordinary differential equations (ODEs). In such cases, some classes of exact solutions can be obtained in explicit form being paramterized by integration constants (see the monographs $[37,38]$ for reviews of some results in GR). At present, it is already possible to elaborate geometric techniques [23-29], the so-called anholonomic frame deformation method (AFDM), for decoupling and generating solutions of PDEs which involve an ansatz resulting in ODEs. We can construct more general classes of exact solutions in MGTs and GR depending generically on two, three and four variables in a "less symmetric" form for four-dimensional (4-d), and extra-dimensional models using advanced geometric, analytic and numerical methods.

The main goal of this article is to apply the AFDM for constructing exact off-diagonal solutions corresponding to cosmological models of MGTs of type $f\left(R, T, R_{\mu \nu} T^{\mu \nu}\right)$ and to study the conditions under which such configurations can be alternatively modeled as effective Einstein spaces with nontrivial off-diagonal 
parametric vacuum and non-vacuum configurations. There will be studied analogous FLRW cosmological dynamics and a reconstruction procedure of the $\Lambda$ CDM universe. We shall not work with exotic anisotropic fluid configurations as in [11-14,20-22], but rather with off-diagonal deformations of de Sitter solutions [15-19]. The problem of matter instabilities in MGTs and GR will be analyzed considering solutions describing nonholonomic cosmological configurations.

\section{Off-diagonal interactions in modified gravity and cosmology}

\subsection{Off-diagonal metrics modelling dark energy}

In a spatially flat spacetime, we can consider the diagonal quadratic form

$$
d s^{2}=\stackrel{\circ}{g}_{\alpha}(t)\left(d u^{\alpha}\right)^{2}=\stackrel{\circ}{a}^{2}(t)\left[\left(d x^{1}\right)^{2}+\left(d x^{2}\right)^{2}+\left(d y^{3}\right)^{2}\right]-d t^{2},
$$

for local coordinates $u^{\alpha}=\left(x^{i}, y^{3}, y^{4}=t\right)$, when $i=1,2$. The FLRW equations are $\frac{3}{\kappa^{2}} \stackrel{\circ}{H}^{2}=\stackrel{\circ}{\rho}$ and $\stackrel{\circ}{\rho}^{\diamond}+3 \stackrel{\circ}{H}(\stackrel{\circ}{\rho}+\stackrel{\circ}{p})=0$, where $\stackrel{\circ}{\rho}$ and $\stackrel{\circ}{p}$ are, respectively, the total energy and pressure of a perfect fluid (pressureless or just radiation), $\stackrel{H}{H}:=\stackrel{\circ}{ }{ }^{\diamond} / \stackrel{\circ}{a}$ for $\stackrel{\circ}{a}^{\diamond}:=\partial \stackrel{\circ}{a} / \partial t=\partial_{4} \stackrel{\circ}{a}=\partial_{t} \stackrel{\circ}{a}$. The constant $\kappa^{2}$ is related to the gravitational (Newton) constant. ${ }^{1}$ Various models have been studied (see reviews and references in [1-10]), in order to explain the observational data of an accelerating universe, and dark energy, $\mathrm{DE}$, and effective, real and/or exotic matter with an equation of state (EoS) of phantom kind, $p=\varpi \rho$, with $\varpi<-1$.

The simplest model of phantom DE is given by $\frac{3}{\kappa^{2}} H_{D E}^{2}=\rho_{D E}$ and $\rho_{D E}^{\diamond}+$ $3 H_{D E}(1+\varpi) \rho_{D E}=0$, which for $\varpi<-1$ admits an exact solution

$$
H_{D E}=\frac{2}{3(1+\varpi)\left(t_{s}-t\right)} .
$$

This solution has a finite-time future singularity (Big Rip) at $t=t_{s}$.

More general models have been elaborated for the Hubble function $H(t)$ determined by a phantom DE coupled with DM, via a coupling constant, $Q$, and the conservation law

$$
\rho_{D E}^{\diamond}+3 H(1+\varpi) \rho_{D E}=-Q \rho_{D E}, \stackrel{\diamond}{\rho_{D M}}+3 H \rho_{D M}=Q \rho_{D M} .
$$

The solutions are $\rho_{D E}={ }^{0} \rho_{D E} e^{-3(1+\varpi)} e^{-Q t}$ and $\rho_{D M} a^{3}=Q^{0} \rho_{D E} \int^{t} d t^{\prime} e^{-3 \varpi} e^{-Q t}$, respectively, where ${ }^{0} \rho_{D E}$ is an integration constant and the EOS is taken to be $p=\varpi \rho_{D E}$. These functions can be used for the second FLRW equation, $-\frac{1}{\kappa^{2}}\left(2 H^{\diamond}+3 H^{2}\right)=p$. We have the solution

$$
H=-Q / 3(1+\varpi)
$$

\footnotetext{
1 We have to introduce a system of notations which is different from that in standard cosmology. This will be convenient for constructing cosmological models with generic off-diagonal metrics.
} 
which corresponds to the de Sitter space evolution, $a(t)=a_{0} e^{-Q t / 3(1+\varpi)}$, where $a_{0}$ is determined from $a_{0}^{3(1+\varpi)}=-\frac{3 \kappa^{2}}{Q^{2}}(1+\varpi)^{2} \varpi{ }^{0} \rho_{D E}$. The value of $H$ in (2) is positive for $\varpi<-1$. This does not mean that the Big Rip singularity in (1) can be avoided, but just shows that the coupling of the phantom DE and DM gives a possibility that the universe could evolve in the de Sitter phase. The first FLRW equation, $\frac{3}{\kappa^{2}} H^{2}=$ $\rho_{D E}+\rho_{D M}$, imposes the relation $\rho_{D M}=(1+\varpi) \rho_{D E}$. We can consider a de Sitter solution as an attractor, with $\varpi \sim-4 / 3$, i.e. $-(1+\varpi) \sim 1 / 3$, which is almost independent from the initial condition; we get a solution of the so-called coincidence problem. If DE does not couple with DM, $\rho_{D M} \sim a^{-3}$ and $\rho_{D E} \sim a^{-3(1+\varpi)}$. The observed $1 / 3$ ratio of $\mathrm{DE}$ and DM is not satisfied which results in a coincidence problem.

Since the DE-DM coupling does not always remove the singularity and there is no such fluid with constant EoS parameter, models were considered which are proportional to a power of the scalar curvature, for instance, $p_{\text {fluid }} \propto R^{1+\epsilon}$, for $\epsilon>0$, and the total EoS parameter is greater than -1 . A Big Rip does not occur for large curvature. Two variants of theories have been exploited where this kind of inhomogeneous effective fluid matter is realized. A conformal anomaly and other quantum effects or by some modified model of gravity, for instance, when the gravitational Lagrange density $R \rightarrow f(R)=R+R^{\varkappa}$. For $1<\varkappa<2$, we have that solutions with $H t \sim-\frac{(\varkappa-1)(2 \varkappa-1)}{\varkappa-2}$ and $w_{\text {eff }} \sim-1-2 H^{\diamond} / 3 H^{2}>-1$ do not result in a Big Rip or any other kind of future singularity, see similar classical and quantum arguments as motivations to study $f$-gravity in Refs. [1-14,20-22,39,40].

Various classes of off-diagonal solutions were studied which can be constructed by geometric methods in MGTs [15-19,23-29,41,42]. Let us briefly recall the main ideas supporting such an approach. Via frame transforms, any metric can be parameterized by off-diagonal ansätze for metrics,

$$
g_{\underline{\alpha} \underline{\beta}}=\left[\begin{array}{cccc}
g_{1}+\omega^{2}\left(w_{1}^{2} h_{3}+n_{1}^{2} h_{4}\right) & \omega^{2}\left(w_{1} w_{2} h_{3}+n_{1} n_{2} h_{4}\right) & \omega^{2} w_{1} h_{3} & \omega^{2} n_{1} h_{4} \\
\omega^{2}\left(w_{1} w_{2} h_{3}+n_{1} n_{2} h_{4}\right) & g_{2}+\omega^{2}\left(w_{2}^{2} h_{3}+n_{2}^{2} h_{4}\right) & \omega^{2} w_{2} h_{3} & \omega^{2} n_{2} h_{4} \\
\omega^{2} w_{1} h_{3} & \omega^{2} w_{2} h_{3} & \omega^{2} h_{3} & 0 \\
\omega^{2} n_{1} h_{4} & \omega^{2} n_{2} h_{4} & 0 & \omega^{2} h_{4}
\end{array}\right],
$$

where the coefficients are functions of type: $g_{1}=g_{2} \sim e^{\psi\left(x^{i}\right)}$ and $n_{i}\left(x^{k}\right)$ (we can fix certain constants for corresponding classes of generating, $\Phi\left(x^{k}, t\right)$, and integration functions), $h_{a}\left[\Phi\left(x^{k}, t\right)\right] \sim h_{a}(t)$, [for $\left.a=3,4\right], w_{i}\left[\Phi\left(x^{k}, t\right)\right] \sim w_{i}(t)$ and $\omega\left(x^{k}, t\right) \sim \omega(t)$, were found to generate exact (in general, nonhomogeneous) cosmological solutions in modified gravity theories. Such generic off-diagonal metrics ${ }^{2}$ can be represented as

$$
d s^{2}=a^{2}(t)\left[\left(e^{1}\right)^{2}+\left(e^{2}\right)^{2}\right]+a^{2}(t) \widehat{h}_{3}(t)\left(\widehat{\mathbf{e}}^{3}\right)^{2}+\left(\widehat{\mathbf{e}}^{4}\right)^{2},
$$

with respect to $\mathrm{N}$-adapted frames $\widehat{\mathbf{e}}^{3}=d y^{3}+n_{i} d x^{i}$ and $\widehat{\mathbf{e}}^{4}=d t+w_{i}(t) d x^{i}$.

2 Which cannot be diagonalized by coordinate transformations. 
We can formulate certain well-defined conditions, see Sect. 3, when off-diagonal deformations $\stackrel{\circ}{g}_{\alpha}(t) \rightarrow g_{\underline{\alpha} \beta}\left(x^{k}, t\right) \sim g_{\underline{\alpha} \beta}(t)$ define new classes of cosmological models. Such deformations mimic contributions from $f$-gravity encoded into the data for $\omega(t), w_{i}(t)$ etc. when corresponding formulas are nonlinear functionals relating generating functions to the (effective) matter sources. The off-diagonal configurations are equivalently modeled as solutions of some effective field equations $\check{\mathbf{R}}_{\beta}^{\alpha}=\check{\Lambda} \delta_{\beta}^{\alpha}$. In result, various classes of cosmological solutions in MGTs can be alternatively modeled by metrics of type (3). In all cases, the scaling factor $a(t)$ is nonlinearly determined by the coefficients $w_{i}(t)$ and $h_{a}(t)$ via a generating function $\Phi(t)$ and an effective source $\Upsilon(t)$. It is possible to model $\Lambda$ CDM cosmology and analogously DE and DM effects with $\rho_{D E}+\rho_{D M}$ encoded into $\Phi(t)$ and $\Upsilon(t)$, but with respect to the adapted frames $\widehat{\mathbf{e}}^{a}(t)$. Solutions with small off-diagonal deformations of metrics may be interpreted in accordance with observational data if the factor $a(t)$ is chosen to be determined nonlinearly/ parameterically, for instance, an effective $H(t)(2)$ with cosmological evolution from a spacetime background encoding $f(R)$-modifications.

Let us suppose that we have found a cosmological solution (3) in a given MGT and analyze how this metric can be formally diagonalized for deformations with a small real parameter $\varepsilon$ (when $0 \leq \varepsilon \ll 1)$. We can consider "homogeneous" approximations of type $\widehat{h}_{3}(t) \approx 1+\varepsilon \widehat{\chi}_{3}(t), w_{i}(t) \sim \varepsilon \check{w}_{i}(t)$ and $n_{i} \sim \varepsilon \check{n}_{i}$ (On inhomogeneity effects in cosmology and possible physical models, see [43]). In a more general context, it is possible to elaborate on "small" local anisotropic deformations depending on space like coordinates when $\widehat{\chi}_{3}\left(x^{k}, t\right), w_{i}(t) \sim \varepsilon \check{w}_{i}\left(x^{k}, t\right)$ and $n_{i} \sim \varepsilon \check{n}_{i}\left(x^{k}\right)$. Some amount of anisotropy is compatible with observational data in various gravity and cosmological theories (see [44,45], for reviews of various approaches related to GR and generalizations of Bianchi, Kasner and Gödel type configurations; [41,42], for off-diagonal configurations; and [46], for $f$-gravity theories). We note also that the approximation $\widehat{h}_{3}(t) \approx 1+\varepsilon \widehat{\chi}_{3}(t)$ can be very restrictive-one can consider more general classes of solutions with arbitrary $\widehat{h}_{3}(t)$.

The metrics with small off-diagonal deformations on $\varepsilon$ and rescaling $\stackrel{a}{a}(t) \rightarrow a(t)$, can be written as

$d s^{2}=a^{2}(t)\left[\left(e^{1}\right)^{2}+\left(e^{2}\right)^{2}\right]+a^{2}(t)\left[1+\varepsilon \widehat{\chi}_{3}(t)\right]\left(d y^{3}+\varepsilon \check{n}_{i} d x^{i}\right)^{2}+\left(d t+\varepsilon \check{w}_{i}(t) d x^{i}\right)^{2}$.

Below, we shall discuss how is it possible to construct subclasses of off-diagonal configurations in a $\widehat{\mathbf{f}}(\widehat{\mathbf{R}}, \ldots)$ gravity where $\widehat{\boldsymbol{\Upsilon}})$ goes into $\check{\Lambda}_{\text {and }} \check{\Phi}^{2}=\check{\Lambda}^{-1}\left[\widehat{\Phi}^{2}|\widehat{\boldsymbol{\Upsilon}}|+\right.$ $\left.\int d \zeta \widehat{\Phi}^{2} \partial_{\zeta}|\widehat{\boldsymbol{\Upsilon}}|\right]$ results in $\widehat{\mathbf{f}} \rightarrow \check{\mathbf{f}}=\check{\mathbf{R}}$, with an effective $\check{\mathbf{R}}_{\beta}^{\alpha}=\check{\Lambda}^{\alpha}{ }_{\beta}^{\alpha}$. We will be able to reproduce the $\Lambda$ CDM model provided the metric (4) defines certain classes of solutions constructed for a corresponding effective action in GR, namely

$$
S=\frac{1}{\kappa^{2}} \int \delta^{4} u \sqrt{\left|{ }^{\varepsilon} \mathbf{g}_{\alpha \beta}\right|}\left({ }^{\varepsilon} \check{\mathbf{R}}-2 \check{\Lambda}+{ }_{m} \mathcal{L}\left({ }^{\varepsilon} \mathbf{g}_{\alpha \beta},{ }_{m} \Psi\right)\right) .
$$

The Ricci scalar ${ }^{\varepsilon} \check{\mathbf{R}}=\check{\mathbf{R}}(a, \varepsilon)$ is constructed for ${ }^{\varepsilon} \mathbf{g}_{\alpha \beta}$ with coefficients of (4), $\check{\Lambda}$ is an effective cosmological constant used for nonholonomic deformations, and ${ }_{m} \mathcal{L}$ is considered for certain effective matter fields with certain pressure ${ }_{m} p$ and energydensity $m \rho$. The EoS are chosen, for simplicity, to correspond to an effective de Sitter 
configuration determined by $\check{\Lambda}$, where $\check{\varpi}:=\check{p}_{\Lambda} / \check{\rho}_{\Lambda}=-1$, with pressure $\check{p}_{\Lambda}$ and energy-density $\check{\rho}_{\Lambda}$.

We can describe the theories determined by (5) and (4) with respect to nonholonomic (non-integrable) dual frames $\widehat{\mathbf{e}}^{\alpha}=\left(e^{i}, \widehat{\mathbf{e}}^{a}\right)$, which is convenient for constructing offdiagonal solutions, or to redefine the constructions with respect to local coordinate coframes $d u^{\alpha}=\left(d x^{i}, d y^{a}\right)$, where certain analogs of the FLRW metric and $\triangle$ CDM like theories can be analyzed. For $\varepsilon \rightarrow 0$, the metric (4) transforms into

$$
d s^{2}=a^{2}(t)\left[\left(e^{1}\right)^{2}+\left(e^{2}\right)^{2}+\left(d y^{3}\right)\right]^{2}+d t^{2},
$$

which is just the FLRW metric but with a re-scaled factor because of $\widehat{\Upsilon} \rightarrow \check{\Lambda}$ and $\widehat{\Phi} \rightarrow \check{\Phi}$.

The corresponding effective Einstein equations with respect to the nonholonomic frames are

$$
3 H^{2}=\kappa_{m}^{2} \rho+\check{\Lambda}, 2 H^{\diamond}=-\kappa^{2}\left({ }_{m} \rho+{ }_{m} P+\check{\Lambda}\right),
$$

where $H^{\diamond}:=a^{\diamond} / a$. We can express ${ }^{\varepsilon} \check{\mathbf{R}}+{ }_{m} \mathcal{L}={ }^{a} \check{\mathbf{R}}+{ }_{m}{ }_{m} \mathcal{L}+\varepsilon{ }_{m}^{1} \mathcal{L}$, where ${ }^{a} \check{\mathbf{R}}$ and ${ }_{m}^{0} \mathcal{L}$ are computed for the metric (6) and ${ }_{m}^{1} \mathcal{L}$ include all $\varepsilon$-deformations in (5). The term ${ }_{m}^{1} \mathcal{L}$ results in the effective splitting ${ }_{m} \rho={ }_{m}^{0} \rho+\varepsilon_{m}^{1} \rho$ and ${ }_{m} p={ }_{m}^{0} p+\varepsilon_{m}^{1} p$. In this way, we can encode the off-diagonal components as certain additional terms into the matter source, or either consider them as a polarization of the effective cosmological constant $\Lambda:=\breve{\Lambda}+\varepsilon^{1} \breve{\Lambda}$. We do not provide explicit formulas for the corrections proportional to $\varepsilon$ because, in the end, we shall take smooth limits $\varepsilon \rightarrow 0$. The main constructions for nonholonomic off-diagonal transforms are based on rescaling $\stackrel{\circ}{a}(t) \rightarrow a^{2}(t)$ generated by the solutions with $\widehat{\Upsilon} \rightarrow \breve{\Lambda}$ and $\widehat{\Phi} \rightarrow \breve{\Phi}$. Possible small inhomogeneous and locally anisotropic contributions, and concordance with observational data, can be estimated similarly to those presented, e.g., in [46]. In coordinate frames, Eq. (7) are written as $3 H^{2}=\kappa_{m}^{20} \rho+\Lambda$, and $2 H^{\diamond}=-\kappa^{2}\left({ }_{m}^{0} \rho+{ }_{m}^{0} P+\Lambda\right)$. For $\varepsilon \rightarrow 0$, the diagonalized solutions are determined by $a$ (and not by $\stackrel{\circ}{a}$ ) and can be parameterized to define and effective $\Lambda \mathrm{CDM}$ like model where $a=a_{c} e^{H_{c} t}$, for a positive constant $a_{c}$. Thus, MGTs with equivalent off-diagonal encodings of $f$-gravity seem to result in realistic cosmological models, at least for small parametric $\varepsilon$-deformations.

We conclude that within certain assumptions, various possible $f(R)$-nonlinear modifications can be encoded into off-diagonal terms and some effective $a(t)$, $\widehat{h}_{3}(t), w_{i}(t)$ via nonlinear interactions. This can be done for more general classes of cosmological solutions with nonlinear gravitational interactions restructuring the spacetime aether before considering certain small $\varepsilon$-parameters.

\subsection{Geometric preliminaries}

We consider a pseudo-Riemannian manifold $V, \operatorname{dim} V=n+m,(n, m \geq 2)$. A Whitney sum $\mathbf{N}$ is defined for its tangent space $T V$, when $\mathbf{N}: T V=h T V \oplus v T V$. This states a nonholonomic (equivalently, non-integrable, or anholonomic) horizontal (h) and vertical (v) splitting, or a nonlinear connection ( $N$-connection) structure. In local form, it is determined by its coefficients $\mathbf{N}=\left\{N_{i}^{a}(u)\right\}$, when $\mathbf{N}=N_{i}^{a}(x, y) d x^{i} \otimes$ 
$\partial / \partial y^{a}$ for certain local coordinates $u=(x, y)$, or $u^{\alpha}=\left(x^{i}, y^{a}\right)$, and $h$-indices $i, j, \ldots=1,2, \ldots, n$ and $v$-indices $a, b, \ldots=n+1, n+2, \ldots, n+m .^{3}$ Such a $h-v$ decomposition can be naturally associated with some $\mathrm{N}$-adapted frame or, respectively, dual frame structures, $\mathbf{e}_{v}=\left(\mathbf{e}_{i}, e_{a}\right)$ and $\mathbf{e}^{\mu}=\left(e^{i}, \mathbf{e}^{a}\right)$,

$\mathbf{e}_{i}=\partial / \partial x^{i}-N_{i}^{a}(u) \partial / \partial y^{a}, e_{a}=\partial_{a}=\partial / \partial y^{a}$, and $e^{i}=d x^{i}, \mathbf{e}^{a}=d y^{a}+N_{i}^{a}(u) d x^{i}$.

The nonholonomy relations hold $\left[\mathbf{e}_{\alpha}, \mathbf{e}_{\beta}\right]=\mathbf{e}_{\alpha} \mathbf{e}_{\beta}-\mathbf{e}_{\beta} \mathbf{e}_{\alpha}=W_{\alpha \beta}^{\gamma} \mathbf{e}_{\gamma}$, with nontrivial anholonomy coefficients $W_{i a}^{b}=\partial_{a} N_{i}^{b}, W_{j i}^{a}=\Omega_{i j}^{a}=\mathbf{e}_{j}\left(N_{i}^{a}\right)-\mathbf{e}_{i}\left(N_{j}^{a}\right)$. The coefficients $\Omega_{i j}^{a}$ define the $\mathrm{N}$-connection curvature.

Any metric structure $\mathbf{g}$ on $\mathbf{V}$ (for physical applications, we consider the signature $(+,+,+,-))$ can be written in two equivalent ways: (1) with respect to a dual local coordinate basis,

$$
\mathbf{g}=\underline{g}_{\alpha \beta} d u^{\alpha} \otimes d u^{\beta},
$$

where $\underline{g}_{\alpha \beta}=\left[\begin{array}{cc}g_{i j}+N_{i}^{a} N_{j}^{b} g_{a b} & N_{j}^{e} g_{a e} \\ N_{i}^{e} g_{b e} & g_{a b}\end{array}\right]$, or (2) as a distinguished metric (in brief, $d$-metric, i.e. in $\mathrm{N}$-adapted form,

$$
\mathbf{g}=g_{\alpha \beta}(u) \mathbf{e}^{\alpha} \otimes \mathbf{e}^{\beta}=g_{i}\left(x^{k}\right) d x^{i} \otimes d x^{i}+g_{a}\left(x^{k}, y^{b}\right) \mathbf{e}^{a} \otimes \mathbf{e}^{a} .
$$

A linear connection is called distinguished, d-connection, $\mathbf{D}=(h D, v D)$, if it preserves under parallelism a prescribed $\mathrm{N}$-connection splitting. Any $\mathbf{D}$ defines an operator of covariant derivation, $\mathbf{D}_{\mathbf{X}} \mathbf{Y}$, for a d-vector field $\mathbf{Y}$ in the direction of a dvector $\mathbf{X}$. We note that any vector $Y(u) \in T \mathbf{V}$ can be parameterized as a d-vector, $\mathbf{Y}=$ $\mathbf{Y}^{\alpha} \mathbf{e}_{\alpha}=\mathbf{Y}^{i} \mathbf{e}_{i}+\mathbf{Y}^{a} e_{a}$, or $\mathbf{Y}=(h Y, v Y)$, with $h Y=\left\{\mathbf{Y}^{i}\right\}$ and $v Y=\left\{\mathbf{Y}^{a}\right\}$, where the $\mathrm{N}$-adapted base vectors and duals, or covectors, are chosen in $\mathrm{N}$-adapted form (8). The local coefficients of $\mathbf{D}_{\mathbf{X}} \mathbf{Y}$ can be computed for $\mathbf{D}=\left\{\boldsymbol{\Gamma}_{\alpha \beta}^{\gamma}=\left(L_{j k}^{i}, L_{b k}^{a}, C_{j c}^{i}, C_{b c}^{a}\right)\right\}$ and h-v-components of $\mathbf{D}_{\mathbf{e}_{\alpha}} \mathbf{e}_{\beta}:=\mathbf{D}_{\alpha} \mathbf{e}_{\beta}$ using $\mathbf{X}=\mathbf{e}_{\alpha}$ and $\mathbf{Y}=\mathbf{e}_{\beta}$. The terms dvector, d-tensor, etc. are used for any vector, tensor valued with coefficients defined in a $\mathrm{N}$-adapted form with respect to the necessary types of tensor products of N-elongate bases and necessary $h$ - $v$-decompositions. We can define three fundamental geometric objects: the d-torsion, $\mathcal{T}$, the non-metricity, $\mathcal{Q}$, and the d-curvature, $\mathcal{R}$, respectively defined by

$$
\begin{aligned}
\mathcal{T}(\mathbf{X}, \mathbf{Y}) & :=\mathbf{D}_{\mathbf{X}} \mathbf{Y}-\mathbf{D}_{\mathbf{Y}} \mathbf{X}-[\mathbf{X}, \mathbf{Y}], \mathcal{Q}(\mathbf{X}):=\mathbf{D}_{\mathbf{X}} \mathbf{g}, \mathcal{R}(\mathbf{X}, \mathbf{Y}) \\
& :=\mathbf{D}_{\mathbf{X}} \mathbf{D}_{\mathbf{Y}}-\mathbf{D}_{\mathbf{Y}} \mathbf{D}_{\mathbf{X}}-\mathbf{D}_{[\mathbf{X}, \mathbf{Y}]} .
\end{aligned}
$$

The N-adapted coefficients, $\mathcal{T}=\left\{\mathbf{T}_{\alpha \beta}^{\gamma}=\left(T^{i}{ }_{j k}, T_{j a}^{i}, T_{j i}^{a}, T_{b i}^{a}, T_{b c}^{a}\right)\right\}, \mathcal{Q}=\left\{\mathbf{Q}_{\alpha \beta}^{\gamma}\right\}$ and $\mathcal{R}=\left\{\mathbf{R}_{\beta \gamma \delta}^{\alpha}=\left(R_{h j k}^{i}, R_{b j k}^{a}, R_{h j a}^{i}, R_{b j a}^{c}, R_{h b a}^{i}, R_{b e a}^{c}\right)\right\}$, of such fundamental geometric objects are computed by introducing $\mathbf{X}=\mathbf{e}_{\alpha}$ and $\mathbf{Y}=\mathbf{e}_{\beta}$, and $\mathbf{D}=\left\{\boldsymbol{\Gamma}_{\alpha \beta}^{\gamma}\right\}$ into the formulas above (see [23-29] for details).

3 The Einstein rule on index summation will be applied if the contrary is not stated. Boldface letters are used in order to emphasize that an $\mathrm{N}$-connection spitting is considered on a spacetime manifold $\mathbf{V}=(V, \mathbf{N})$. 
A d-connection $\mathbf{D}$ is compatible with a d-metric $\mathbf{g}$ if and only if $\mathcal{Q}=\mathbf{D g}=0$. Any metric structure $\mathbf{g}$ on $\mathbf{V}$ is characterized by a unique metric compatible and torsionless linear connection called the Levi-Civita (LC) connection, $\nabla$. It should be noted that $\nabla$ is not a d-connection because it does not preserve under parallelism the $\mathrm{N}$-connection splitting. Nevertheless, such a $h-v$ decomposition allows us to define $\mathrm{N}$-adapted distortions of any d-connection $\mathbf{D}$,

$$
\mathbf{D}=\nabla+\mathbf{Z}
$$

with respective conventional "non-boldface" and "boldface" symbols for the coefficients: $\nabla=\left\{\Gamma_{\beta \gamma}^{\alpha}\right\}$ and, for the distortion d-tensor, $\mathbf{Z}=\left\{\mathbf{Z}_{\beta \gamma}^{\alpha}\right\}$.

This stands for any prescribed $\mathbf{N}$ and $\mathbf{g}=h \mathbf{g}+v \mathbf{g}$, but alternatively to $\nabla$, on $\mathbf{V}$, we can work with the so-called canonical d-connection, $\widehat{\mathbf{D}}$, when

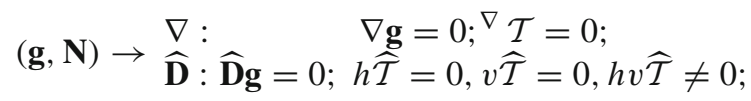

are completely defined by the same metric structure. The canonical distortion d-tensor $\widehat{\mathbf{Z}}$ in the distortion relation of type (11), $\widehat{\mathbf{D}}=\nabla+\widehat{\mathbf{Z}}$, is an algebraic combination of the coefficients of the corresponding torsion d-tensor $\widehat{\mathcal{T}}=\left\{\widehat{\mathbf{T}}_{\beta \gamma}^{\alpha}\right\}$. The respective coefficients of the torsions, $\widehat{\mathcal{T}}$ and ${ }^{\nabla} \mathcal{T}=0$, and curvatures, $\widehat{\mathcal{R}}=\left\{\widehat{\mathbf{R}}_{\beta \gamma \delta}^{\alpha}\right\}$ and $\nabla_{\mathcal{R}}=\left\{R_{\beta \gamma \delta}^{\alpha}\right\}$, of $\widehat{\mathbf{D}}$ and $\nabla$ can be defined and computed using standard formulas. The coefficients $\widehat{\mathbf{T}}_{\beta \gamma}^{\alpha}$ are not trivial but nonholonomically induced by anholonomy coefficients $W_{\alpha \beta}^{\gamma}$ and certain off-diagonal coefficients of the metric.

The Ricci tensors of $\widehat{\mathbf{D}}$ and $\nabla$ are computed in the standard form, $\widehat{\mathcal{R}} i c=\left\{\widehat{\mathbf{R}}_{\beta \gamma}:=\right.$ $\left.\widehat{\mathbf{R}}_{\alpha \beta \gamma}^{\gamma}\right\}$ and Ric $=\left\{R_{\beta \gamma}:=R_{\alpha \beta \gamma}^{\gamma}\right\}$. With respect to N-adapted coframes (8), the Ricci d-tensor $\widehat{\mathcal{R}} i c$ is characterized by four $h-v$ N-adapted coefficients

$$
\widehat{\mathbf{R}}_{\alpha \beta}=\left\{\widehat{R}_{i j}:=\widehat{R}_{i j k}^{k}, \widehat{R}_{i a}:=-\widehat{R}_{i k a}^{k}, \widehat{R}_{a i}:=\widehat{R}_{a i b}^{b}, \widehat{R}_{a b}:=\widehat{R}_{a b c}^{c}\right\},
$$

and (an alternative to $R:=\mathbf{g}^{\alpha \beta} R_{\alpha \beta}$ ) scalar curvature, $\widehat{\mathbf{R}}:=\mathbf{g}^{\alpha \beta} \widehat{\mathbf{R}}_{\alpha \beta}=g^{i j} \widehat{R}_{i j}+$ $g^{a b} \widehat{R}_{a b}$.

We emphasize that any (pseudo) Riemannian geometry can be equivalently described by both geometric data $(\mathbf{g}, \nabla)$ and $(\mathbf{g}, \mathbf{N}, \widehat{\mathbf{D}})$. For instance, there are canonical distortion relations $\widehat{\mathcal{R}}={ }^{\nabla} \mathcal{R}+{ }^{\nabla} \mathcal{Z}$ and $\widehat{\mathcal{R}} i c=R i c+\widehat{\mathcal{Z}} i c$, where the respective distortion d-tensors $\nabla \mathcal{Z}$ and $\widehat{\mathcal{Z}} i c$ are computed by introducing $\widehat{\mathbf{D}}=\nabla+\widehat{\mathbf{Z}}$ into the corresponding formulas for curvature and (12). The canonical data $(\mathbf{g}, \mathbf{N}, \widehat{\mathbf{D}})$ provide an example of nonholonomic (pseudo-) Riemannian manifold which is a standard one but enabled with a nonholonomic distribution determined by $(\mathbf{g}, \mathbf{N})$. If the coefficients $\Omega_{i j}^{a}=0$, such a distribution is holonomic, i.e. integrable. Nevertheless, physical theories formulated in terms of data as $(\mathbf{g}, \nabla)$, or $(\mathbf{g}, \mathbf{N}, \widehat{\mathbf{D}})$, are not equivalent if certain additional conditions are not imposed.

We can introduce the Einstein d-tensor of $\widehat{\mathbf{D}}, \widehat{\mathbf{E}}_{\alpha \beta}:=\widehat{\mathbf{R}}_{\alpha \beta}-\frac{1}{2} \mathbf{g}_{\alpha \beta} \widehat{\mathbf{R}}$, and construct a $\mathrm{N}$-adapted energy momentum tensor for a Lagrange density ${ }^{m} \mathcal{L}$ of the matter 
fields, $\widehat{\mathbf{T}}_{\alpha \beta}:=-\frac{2}{\sqrt{\left|\mathbf{g}_{\mu \nu}\right|}} \frac{\delta\left(\sqrt{\left|\mathbf{g}_{\mu v}\right|}{ }^{m} \widehat{\mathcal{L}}\right)}{\delta \mathbf{g}^{\alpha \beta}}$, performing a N-adapted variational calculus with respect to frames (8), and considering that $\widehat{\mathbf{D}}$ is used as covariant derivative instead of $\nabla$. A nonholonomic deformation of Einstein's gravity is constructed, being $\nabla \rightarrow \widehat{\mathbf{D}}=\nabla+\widehat{\mathbf{Z}}$, with gravitational field equations

$$
\widehat{\mathbf{R}}_{\alpha \beta}=\kappa^{2}\left(\widehat{\mathbf{T}}_{\alpha \beta}-\frac{1}{2} \mathbf{g}_{\alpha \beta} \widehat{\mathbf{T}}\right)
$$

for a conventional gravitational constant $\kappa^{2}$ and $\widehat{\mathbf{T}}:=\mathbf{g}^{\mu \nu} \widehat{\mathbf{T}}_{\mu \nu}$. Such equations are different from the standard Einstein equations in GR because, in general, $\widehat{\mathbf{R}}_{\alpha \beta} \neq R_{\alpha \beta}$ and $\widehat{\mathbf{T}}_{\alpha \beta} \neq T_{\alpha \beta}$, where $T_{\alpha \beta}:=-\frac{2}{\sqrt{\left|\mathbf{g}_{\mu \nu}\right|}} \frac{\delta\left(\sqrt{\left|\mathbf{g}_{\mu \nu}\right|}{ }^{m} \mathcal{L}\right)}{\delta \mathbf{g}^{\alpha \beta}}$ for ${ }^{m} \mathcal{L}\left[\mathbf{g}_{\alpha \beta}, \nabla\right] \neq{ }^{m} \widehat{\mathcal{L}}\left[\mathbf{g}_{\alpha \beta}, \widehat{\mathbf{D}}\right]$.

LC-configurations can be extracted from certain classes of solutions of Eq. (13) if additional conditions are imposed, resulting in zero values for the canonical d-torsion, $\widehat{\mathcal{T}}=0$. In $\mathrm{N}$-adapted coefficient form, such conditions are equivalent to

$\widehat{T}^{i}{ }_{j k}=\widehat{L}_{j k}^{i}-\widehat{L}_{k j}^{i}, \widehat{T}^{i}{ }_{j a}=\widehat{C}_{j b}^{i}, \widehat{T}^{a}{ }_{j i}=-\Omega^{a}{ }_{j i}, \widehat{T}_{a j}^{c}=\widehat{L}_{a j}^{c}-e_{a}\left(N_{j}^{c}\right), \widehat{T}_{b c}^{a}=\widehat{C}_{b c}^{a}-\widehat{C}_{c b}^{a}$.

It should be emphasized that we are able to find generic off-diagonal solutions of the Einstein equations in GR depending on three and more coordinates for $\widehat{\mathbf{D}} \rightarrow \nabla$, when $\widehat{\mathbf{R}}_{\alpha \beta} \rightarrow R_{\alpha \beta}$ and $\widehat{\mathbf{T}}_{\alpha \beta} \rightarrow T_{\alpha \beta}$, if the nonholonomic constraints (14) are imposed after certain classes of solutions were found for $\widehat{\mathbf{D}} \neq \nabla$. But we are not able to decouple such systems of nonlinear PDEs if the zero torsion condition for $\nabla$ is imposed from the very beginning.

\subsection{Nonholonomic $f$-modified gravity theories}

Different models of modified gravity are formulated for independent metric and linear connection fields with a corresponding Palatini type variational formulation (see [110]). The gravitational and matter field equations in MGTs consist in very sophisticate systems of nonlinear PDEs for which finding exact solutions is a very difficult technical task, even for the simplest diagonal ansätze with the coefficients of the metrics and connections depending on just one (time or space) variable. Nevertheless, the AFDM [23-29] seems to work efficiently and allows to construct off-diagonal solutions in MGTs and GR [41,42].

Let us consider three classes of equivalent MGTs defined for the same metric field $\mathbf{g}=\left\{g_{\mu \nu}\right\}$ but with different actions (and related functionals) for gravity, ${ }^{g} S$, and matter, ${ }^{m} S$, fields,

$$
\begin{aligned}
& \mathcal{S}={ }^{g} \mathcal{S}+{ }^{m} \mathcal{S}=\frac{1}{2 \kappa^{2}} \int f\left(R, T, R_{\alpha \beta} T^{\alpha \beta}\right) \sqrt{|g|} d^{4} u+\int{ }^{m} \mathcal{L} \sqrt{|g|} d^{4} u \\
& ={ }^{g} \widehat{\mathbf{S}}+{ }^{m} \widehat{\mathbf{S}}=\frac{1}{2 \kappa^{2}} \int \widehat{\mathbf{f}}\left(\widehat{\mathbf{R}}, \widehat{\mathbf{T}}, \widehat{\mathbf{R}}_{\alpha \beta} \widehat{\mathbf{T}}^{\alpha \beta}\right) \sqrt{|\widehat{\mathbf{g}}|} \mathbf{d}^{4} u+\int{ }^{m} \widehat{\mathbf{L}} \sqrt{|\widehat{\mathbf{g}}|} \mathbf{d}^{4} u \\
& ={ }^{g} \check{\mathbf{S}}+{ }^{m} \check{\mathbf{S}}=\frac{1}{2 \kappa^{2}} \int \check{\mathbf{R}} \sqrt{|\check{\mathbf{g}}|} \mathbf{d}^{4} u+\check{\Lambda} \int \sqrt{|\check{\mathbf{g}}|} \mathbf{d}^{4} u \text {. }
\end{aligned}
$$


We use boldface $\mathbf{d}^{4} u$ in order to emphasize that the integration volume is for $\mathrm{N}$ elongated differentials (8), $\kappa^{2}$ is the gravitational coupling constant, the values with " $\wedge$ " are computed for a canonical d-connection $\widehat{\mathbf{D}}$ and the values with " $\vee$ " for re-defined geometric data $(\check{\mathbf{g}}, \check{\mathbf{N}}, \check{\mathbf{D}})$ for certain nonholonomic frame transforms and nonholonomic deformations $g_{\alpha \beta} \sim \widehat{\mathbf{g}}_{\alpha \beta} \sim \check{\mathbf{g}}_{\alpha \beta} .{ }^{4}$ For simplicity, we consider matter actions which only depend on the coefficients of a metric field and not on their derivatives, $\widehat{\mathbf{T}}^{\alpha \beta}={ }^{m} \widehat{\mathbf{L}} \widehat{\mathbf{g}}^{\alpha \beta}+2 \delta\left({ }^{m} \widehat{\mathbf{L}}\right) / \delta \widehat{\mathbf{g}}_{\alpha \beta}$.

We assume that the matter content of the universe can be approximated by a perfect fluid,

$$
\widehat{\mathbf{T}}_{\alpha \beta}=p \widehat{\mathbf{g}}_{\alpha \beta}+(\rho+p) \widehat{\mathbf{v}}_{\alpha} \widehat{\mathbf{v}}_{\beta}
$$

is defined for certain (effective) energy and pressure densities, respectively, $\widehat{\mathbf{v}}_{\alpha}$ being the four-velocity of the fluid for which $\widehat{\mathbf{v}}_{\alpha} \widehat{\mathbf{v}}^{\alpha}=-1$ and $\widehat{\mathbf{v}}^{\alpha}=(0,0,0,1)$ in N-adapted comoving frames/coordinates. Frame transforms of metrics of type $\widehat{\mathbf{g}}_{\alpha \beta}=\mathbf{e}_{\alpha}^{\alpha^{\prime}} \mathbf{e}_{\beta}^{\beta^{\prime}} \stackrel{\stackrel{g}{g}}{\alpha^{\prime} \beta^{\prime}}$, will be studied beginning with the FLRW diagonalized element

$$
\begin{aligned}
d \stackrel{\circ}{s}^{2} & ={\stackrel{\circ}{\alpha^{\prime} \beta^{\prime}}} d u^{\alpha^{\prime}} d u^{\beta^{\prime}}=\stackrel{\circ}{a}^{2}(t)\left[d r^{2}+r^{2} d \theta^{2}+r^{2} \sin ^{2} \theta d \varphi^{2}\right]-d t^{2}, \\
& =\stackrel{\circ}{a}^{2}(t)\left[d x^{2}+d y^{2}+d z^{2}\right]-d t^{2},
\end{aligned}
$$

where the scale factor $\stackrel{\circ}{a}(t)$ (we use also the value $\stackrel{H}{H}:=\stackrel{\circ}{a} / \stackrel{\circ}{a}$, for $\stackrel{\circ}{a}:=d \stackrel{\circ}{a} / d t$ ) with signature $(+,+,+,-)$, and a parametrization of coordinates in the form $u^{\alpha^{\prime}}=\left(x^{1^{\prime}}=\right.$ $\left.r, x^{2^{\prime}}=\theta, y^{3^{\prime}}=\varphi, y^{4^{\prime}}=t\right)$, or as Cartesian coordinates $\left(x^{1^{\prime}}=x, x^{2^{\prime}}=y, y^{3^{\prime}}=\right.$ $\left.z, y^{4^{\prime}}=t\right)$. For such cosmological metrics, the main issues of the Einstein and modified Universes are encoded into energy-momentum tensor $\stackrel{\circ}{T}_{\alpha \beta}=\stackrel{\circ}{p} \stackrel{\circ}{\alpha}_{\alpha \beta}+(\stackrel{\circ}{\rho}+\stackrel{\circ}{p}) \stackrel{\circ}{\alpha}_{\alpha} \stackrel{\circ}{v}_{\beta}$ (we omit primes or other distinctions in the coordinate indices if there is no ambiguity) arising from a matter Lagrangian ${ }^{m} \stackrel{\circ}{\mathcal{L}}$ for $\stackrel{\circ}{T}_{\beta}^{\alpha}=\operatorname{diag}[0,0,0,-\stackrel{\circ}{\rho}]$ with

$$
\stackrel{\circ}{T}(t)=\stackrel{\circ}{T}_{\alpha}^{\alpha}=-\stackrel{\circ}{\rho}, \stackrel{\circ}{P}(t)=\stackrel{\circ}{R}_{\alpha \beta} \stackrel{\circ}{T}^{\alpha \beta}=\stackrel{\circ}{R}_{44} \stackrel{\circ}{T}^{44}=-3 \stackrel{\circ}{\rho}\left(\stackrel{\circ}{H}^{2}+\stackrel{\circ}{H}^{\diamond}\right) .
$$

There will constructed nonhomogeneous and locally anisotropic cosmological solutions of type (10) with

$$
\begin{aligned}
g_{i} & =g_{i}\left(x^{k}\right)=\eta_{i}\left(x^{k}, y^{4}\right) \stackrel{\circ}{g}_{i}\left(x^{k}, y^{4}\right)=e^{\psi\left(x^{k}\right)}, \\
g_{a} & =\omega^{2}\left(x^{k}, y^{4}\right) h_{a}\left(x^{k}, y^{4}\right) \\
& =\omega^{2}\left(x^{k}, y^{4}\right) \eta_{a}\left(x^{k}, y^{4}\right) \stackrel{\circ}{g}_{a}\left(x^{k}, y^{4}\right), N_{i}^{3}=n_{i}\left(x^{k}\right), N_{i}^{4}=w_{i}\left(x^{k}, y^{4}\right) .
\end{aligned}
$$

In Eq. (19), there is no summation on repeated indices, $\eta_{\alpha}=\left(\eta_{i}, \eta_{a}\right)$ are polarization functions, the $\mathrm{N}$-connection coefficients are determined by $n_{i}$ and $w_{i}$, the vertical conformal factor $\omega$ may depend on all spacetime coordinates and $\stackrel{\circ}{g}_{\alpha}=\left(\stackrel{\circ}{g}_{i}, \stackrel{\circ}{g}_{a}\right)$ define the "prime" diagonal metric if $\eta_{\alpha}=1$ and $N_{i}^{a}=0$. The "target" off-diagonal metrics are with Killing symmetry on $\partial / \partial y^{3}$ when the coefficients (19) do not depend on $y^{3}$.

4 see details in Sects. 3.1.2 and 3.2. 
We can consider nonholonomic deformations with non-Killing symmetries when, for instance, $\omega\left(x^{k}, y^{4}\right) \rightarrow \omega\left(x^{k}, y^{3}, y^{4}\right)$, which results in a more cumbersome calculus and geometric techniques. For simplicity, we do not study such generalizations in this work (see examples in [23-29]).

The quadratic line element is parameterized

$$
\begin{aligned}
d s^{2}= & a^{2}\left(x^{k}, t\right)\left[\eta_{1}\left(x^{k}, t\right)\left(d x^{1}\right)^{2}+\eta_{2}\left(x^{k}, t\right)\left(d x^{2}\right)^{2}\right]+a^{2}\left(x^{k}, t\right) \widehat{h}_{3}\left(x^{k}, t\right)\left(\widehat{\mathbf{e}}^{3}\right)^{2} \\
& +\omega^{2}\left(x^{k}, t\right) h_{4}\left(x^{k}, t\right)\left(\widehat{\mathbf{e}}^{4}\right)^{2},
\end{aligned}
$$

when $a^{2}\left(x^{k}, t\right) \eta_{i}\left(x^{k}, t\right)=e^{\psi\left(x^{k}\right)}$, for $i=1,2 ; a^{2} \widehat{h}_{3}=\omega^{2}\left(x^{k}, t\right) h_{3}\left(x^{k}, t\right)$, and $\widehat{\mathbf{e}}^{3}=d y^{3}+n_{i}\left(x^{k}\right) d x^{i}, \widehat{\mathbf{e}}^{4}=d y^{4}+w_{i}\left(x^{k}, t\right) d x^{i}$. Functions $\eta_{i}, \eta_{a}, a, \psi, \omega, n_{i}, w_{i}$ will be found such that, via nonholonomic transforms (19), when ${\stackrel{\circ}{\alpha^{\prime}} \beta^{\prime}}(t)(17) \rightarrow$ $\widehat{\mathbf{g}}_{\alpha \beta}\left(x^{k}, t\right)(20)$, off-diagonal nonhomogeneous cosmological solutions are generated in a model of MGT (15). We can consider subclasses of off-diagonal cosmological solutions with deformed symmetries when nontrivial limits $\widehat{\mathbf{g}}_{\alpha \beta}\left(x^{k}, t\right) \rightarrow \widehat{\mathbf{g}}_{\alpha \beta}(t)$ can be found and define viable cosmological models.

Applying an $\mathrm{N}$-adapted variational procedure with respect to nonholonomic bases (8) for the action $\mathcal{S}={ }^{g} \widehat{\mathbf{S}}+{ }^{m} \widehat{\mathbf{S}}$, which is similar to that in [20-22] but for $\nabla \rightarrow \widehat{\mathbf{D}}$ and matter source $\widehat{\mathbf{T}}_{\alpha \beta}$ (16), we obtain the field equations for the corresponding modified gravity theory

$$
\begin{aligned}
& \widehat{\mathbf{R}}_{\alpha \beta}{ }^{1} \widehat{\mathbf{f}}-\frac{1}{2} \widehat{\mathbf{g}}_{\alpha \beta} \widehat{\mathbf{f}}+\left(\widehat{\mathbf{g}}_{\alpha \beta} \widehat{\mathbf{D}}^{\mu} \widehat{\mathbf{D}}_{\mu}-\widehat{\mathbf{D}}_{\alpha} \widehat{\mathbf{D}}_{\beta}\right)^{1} \widehat{\mathbf{f}}+\left(\widehat{\mathbf{T}}_{\alpha \beta}+\boldsymbol{\Theta}_{\alpha \beta}\right)^{2} \widehat{\mathbf{f}} \\
& +\boldsymbol{\Xi}_{\alpha \beta}{ }^{3 \widehat{\mathbf{f}}}+\frac{1}{2}\left(\widehat{\mathbf{D}}^{\mu} \widehat{\mathbf{D}}_{\mu} \widehat{\mathbf{T}}_{\alpha \beta}{ }^{3} \widehat{\mathbf{f}}+\widehat{\mathbf{g}}_{\alpha \beta} \widehat{\mathbf{D}}_{\mu} \widehat{\mathbf{D}}_{\nu} \widehat{\mathbf{T}}^{\mu \nu}{ }^{3} \widehat{\mathbf{f}}\right)-\widehat{\mathbf{D}}_{\nu} \widehat{\mathbf{D}}_{(\alpha} \widehat{\mathbf{T}}_{\beta)}{ }^{3} \widehat{\mathbf{f}}^{2}=\kappa^{2} \widehat{\mathbf{T}}_{\alpha \beta},
\end{aligned}
$$

for $\boldsymbol{\Theta}_{\alpha \beta}=p \widehat{\mathbf{g}}_{\alpha \beta}-2 \widehat{\mathbf{T}}_{\alpha \beta}, \boldsymbol{\Xi}_{\alpha \beta}=2 \widehat{\mathbf{E}}_{(\alpha}^{\nu} \widehat{\mathbf{T}}_{\beta) \nu}-p \widehat{\mathbf{E}}_{\alpha \beta}-\frac{1}{2} \widehat{\mathbf{R T}}_{\alpha \beta}$, with respective d-tensors defined by Eq. (12), where ${ }^{1} \widehat{\mathbf{f}}:=\partial \widehat{\mathbf{f}} / \partial \widehat{\mathbf{R}}, \quad{ }^{2} \widehat{\mathbf{f}}:=\partial \widehat{\mathbf{f}} / \partial \widehat{\mathbf{T}}$ and ${ }^{3} \widehat{\mathbf{f}}:=\partial \widehat{\mathbf{f}} / \partial \widehat{\mathbf{P}}$, when $\widehat{\mathbf{P}}=\widehat{\mathbf{R}}_{\alpha \beta} \widehat{\mathbf{T}}^{\alpha \beta}$ and $(\alpha \beta)$ denotes symmetrization of the indices.

In general, the divergence with $\widehat{\mathbf{D}}$ and/or $\nabla$ of Eq. (21) is not zero. Also Eq. (13) has a similar property. In the last case, we can obtain the continuity equations as in GR and then deform them by using the distortions (11), which for the canonical $\mathrm{d}$-connections are completely determined by the metric structure. There are certain types of conservation laws for matter fields with additional nonholonomic constraints. Remarkably, such sophisticate nonholonomic and nonlinear systems can be solved in very general off-diagonal forms, by applying the anholonomic frame deformation method. In order to compare these results and to find possible applications in modern cosmology, we will consider a particular equation of state $(\operatorname{EoS}) p=\varpi \rho$ with $\varpi=$ const, and study the cosmology of off-diagonal distortions of certain FLRW models considered in the framework of GR and its modifications. In both cases, by exploring some particular classes of solutions, the dynamics of the matter sector of generalized $f\left(R, T, R_{\mu \nu} T^{\mu \nu}\right)$ gravity (with respect to $\mathrm{N}$-adapted frames) may lead to similar cosmological scenarios as GR, but with nonholonomic constraints and deformations. 


\section{The AFDM and exact solutions in MGTs}

A surprising property of Eqs. (13) and (21) is that they can be integrated in very general form with generic off-diagonal metrics when their coefficients depend on all spacetime coordinates via various classes of generating and integration functions and constants. In particular, we can consider such generating and integration functions when $\widehat{\mathbf{g}}_{\alpha \beta}\left(x^{k}, t\right)$ (20) result in off-diagonal metrics of type $\widehat{\mathbf{g}}_{\alpha \beta}(t)$ depending on the parameters and possible (non-) commutative Lie algebra or algebroid symmetries.

\subsection{Off-diagonal FLRW like cosmological models}

We shall study cosmological models with sources of type (16) when the four-velocity $\widehat{\mathbf{v}}_{\alpha}$ is re-parameterized in a way that for some frame transforms as

$$
\begin{aligned}
\widehat{\mathcal{Y}}_{\alpha \beta} & :=\kappa^{2}\left(\widehat{\mathbf{T}}_{\alpha \beta}-\frac{1}{2} \mathbf{g}_{\alpha \beta} \widehat{\mathbf{T}}\right) \\
& \rightarrow \operatorname{diag}\left[\Upsilon_{1}=\Upsilon_{2}, \Upsilon_{2}={ }^{h} \Upsilon\left(x^{i}\right), \Upsilon_{3}=\Upsilon_{4}, \Upsilon_{4}={ }^{v} \Upsilon\left(x^{i}, t\right)\right] \\
& \rightarrow \widehat{\Lambda} \mathbf{g}_{\alpha \beta} \text { (redefining the generating functions and sources), }
\end{aligned}
$$

for effective $h$ - and $v$-polarized sources, respectively, ${ }^{h} \Upsilon\left(x^{i}\right)$ and $\Upsilon_{4}={ }^{v} \Upsilon\left(x^{i}, t\right)$, or an effective cosmological constant $\widehat{\Lambda}$. For simplicity, we can consider effective matter sources and "prime" metrics with Killing symmetry on $\partial / \partial_{3}$, i.e. when the effective matter sources and d-metrics do not depend on $y^{3} .^{5}$ In brief, the partial derivatives $\partial_{\alpha}=\partial / \partial u^{\alpha}$ will be written as $s^{\bullet}=\partial s / \partial x^{1}, s^{\prime}=\partial s / \partial x^{2}, s^{*}=\partial s / \partial y^{3}, s^{\diamond}=$ $\partial s / \partial y^{4}$.

The nontrivial components of the nonholonomic Einstein equations (12), with source (22) parameterized with respect to (co) bases (8), for a d-metric ansätze (10) with coefficients (19), are (see Refs. [20-22])

$$
\begin{aligned}
\psi^{\bullet \bullet}+\psi^{\prime \prime} & =2^{h} \Upsilon \\
\phi^{\diamond} h_{3}^{\diamond} & =2 h_{3} h_{4}{ }^{v} \Upsilon \\
n_{i}^{\diamond \diamond}+\gamma n_{i}^{\diamond} & =0, \\
\beta w_{i}-\alpha_{i} & =0, \\
\partial_{i} \omega-\left(\partial_{i} \phi / \phi^{\diamond}\right) \omega^{\diamond} & =0,
\end{aligned}
$$

for

$$
\alpha_{i}=h_{3}^{\diamond} \partial_{i} \phi, \beta=h_{3}^{\diamond} \phi^{\diamond}, \gamma=\left(\ln \left|h_{3}\right|^{3 / 2} /\left|h_{4}\right|\right)^{\diamond}
$$

where

$$
\phi=\ln \mid h_{3}^{\diamond} / \sqrt{\left|h_{3} h_{4}\right|} \text {, and or } \Phi:=e^{\phi},
$$

\footnotetext{
5 The method can be extended to account for $y^{3}$ dependence and non-Killing configurations (see [23-29]). In this paper the local coordinates and ansätze for d-metrics are parameterized in different forms than in previous works, what is more convenient for the study of cosmological models.
} 
is considered as a generating function. In these formulas, we consider $h_{a}^{\diamond} \neq 0$, ${ }^{h} \Upsilon,{ }^{v} \Upsilon \neq 0$. Formula (28) is a nontrivial solution of (27) with coefficients (29), when

$$
w_{i}=\partial_{i} \phi / \phi^{\diamond}
$$

and $\mathbf{e}_{i} \omega=\partial_{i} \omega-n_{i} \omega^{*}-w_{i} \omega^{\diamond}=0$.

The d-torsion (14) vanishes if the (Levi-Civita, LC) conditions $\widehat{L}_{a j}^{c}=e_{a}\left(N_{j}^{c}\right), \widehat{C}_{j b}^{i}=$ $0, \Omega^{a}{ }_{j i}=0$, are satisfied:

$$
\begin{aligned}
w_{i}^{\diamond} & =\left(\partial_{i}-w_{i} \partial_{4}\right) \ln \sqrt{\left|h_{4}\right|},\left(\partial_{i}-w_{i} \partial_{4}\right) \ln \sqrt{\left|h_{3}\right|}=0, \\
\partial_{k} w_{i} & =\partial_{i} w_{k}, n_{i}^{\diamond}=0, \partial_{i} n_{k}=\partial_{k} n_{i} .
\end{aligned}
$$

The decoupling property of the above system of equations follows from the facts that: (1) integrating the 2-d Laplace Eq. (24) one finds solutions for the $h$-coefficients of the d-metric, and (2) the solutions for the coefficients of the d-metric can be found from (25) and (30). (3) Then the N-connection coefficients $w_{i}$ and $n_{i}$ can be found from (26) and (27), respectively.

\subsubsection{Cosmological solutions with nonholonomically induced torsion}

The Eqs. (24) and (27) can be solved, respectively, for any source ${ }^{h} \Upsilon\left(x^{k}\right)$ and generating function $\phi\left(x^{k}, t\right)$. The system (25) and (30) can be written as $h_{3} h_{4}=\phi^{\diamond} h_{3}^{\diamond} / 2^{v} \Upsilon$ and $\left|h_{3} h_{4}\right|=\left(h_{3}^{\diamond}\right)^{2} e^{-2 \phi}$, for any nontrivial source ${ }^{v} \Upsilon\left(x^{i}, t\right)$ in (25). Introducing the first equation into the second, one finds $\left|h_{3}^{\diamond}\right|=\frac{\left(e^{2 \phi}\right)^{\diamond}}{\left.4\right|^{v} \Upsilon \mid}=\frac{\Phi^{\diamond} \Phi}{\left.2\right|^{v} \Upsilon \mid}$, i.e. $h_{3}={ }^{0} h_{3}\left(x^{k}\right)+\frac{\epsilon_{3} \epsilon_{4}}{4} \int d t \frac{\left(\Phi^{2}\right)^{\diamond}}{{ }^{v} \Upsilon}$, where ${ }^{0} h_{3}\left(x^{k}\right)$ and $\epsilon_{3}, \epsilon_{4}= \pm 1$. Using again the 1 st equation, we get $h_{4}=\frac{\phi^{\diamond}\left(\ln \sqrt{\left|h_{3}\right|}\right)^{\diamond}}{2^{v} \Upsilon}=\frac{1}{2^{v} \Upsilon} \frac{\Phi^{\diamond}}{\Phi} \frac{h_{3}^{\diamond}}{h_{3}}$. We can simplify such formulas for $h_{3}$ and $h_{4}$ if we redefine the generating function, $\Phi \rightarrow \widehat{\Phi}$, where $\left(\Phi^{2}\right)^{\diamond} /{ }^{v} \Upsilon \mid=\left(\widehat{\Phi}^{2}\right)^{\diamond} / \Lambda$, i.e.

$$
\Phi^{2}=\Lambda^{-1}\left[\widehat{\Phi}^{2}\left|{ }^{v} \Upsilon\right|+\left.\left.\int d t \widehat{\Phi}^{2}\right|^{v} \Upsilon\right|^{\diamond}\right]
$$

for an effective cosmological constant $\Lambda$ which may take positive or negative values. We can integrate on $t$, include the integration function ${ }^{0} h_{3}\left(x^{k}\right)$ in $\widehat{\Phi}$ and write

$$
h_{3}[\widehat{\Phi}]=\widehat{\Phi}^{2} / 4 \Lambda \text {. }
$$

Introducing this formula and (33) and that for $h_{4}$, we compute

$$
h_{4}[\widehat{\Phi}]=\frac{(\ln |\Phi|)^{\diamond}}{\left.4\right|^{v} \Upsilon \mid}=\frac{\left(\widehat{\Phi}^{2}\right)^{\diamond}}{8}\left[\left.\left.\widehat{\Phi}^{2}\right|^{v} \Upsilon\left|+\int d t \widehat{\Phi}^{2}\right|^{v} \Upsilon\right|^{\diamond}\right]^{-1}
$$


As next step, we need solve Eq. (26) by integrating on $t$ twice. We obtain

$$
n_{k}={ }_{1} n_{k}+{ }_{2} n_{k} \int d t h_{4} /\left(\sqrt{\left|h_{3}\right|}\right)^{3} \text {, }
$$

where ${ }_{1} n_{k}\left(x^{i}\right), 2 n_{k}\left(x^{i}\right)$ are integration functions and $h_{a}[\widehat{\Phi}]$ are given by formulas (34) and (35). If we fix ${ }_{2} n_{k}=0$, we shall be able to find $n_{k}={ }_{1} n_{k}\left(x^{i}\right)$ which have zero torsion limits (see examples in Sect. 3.1.2).

The solutions of (27) are given by (31), which for different types of generating functions are parameterized

$$
w_{i}=\frac{\partial_{i} \Phi}{\Phi^{\diamond}}=\frac{\partial_{i}\left(\Phi^{2}\right)}{\left(\Phi^{2}\right)^{\diamond}},
$$

where the integral functional $\Phi\left[\widehat{\Phi},{ }^{v} \Upsilon\right]$ is given by (33).

We can introduce certain polarization functions $\eta_{\alpha}$ in order to write the d-metric of such solutions in the form (20). Let us fix $\omega^{2}=\left|h_{4}\right|^{-1}$ to satisfy the condition (28), which for a generating function $\Phi[\phi]$ is equivalent to $\Phi^{\diamond} \partial_{i} h_{4}-\partial_{i} \Phi h_{4}^{\diamond}=0$. These first order PDE equations impose certain conditions on the class of generating function $\Phi$ and source ${ }^{v} \Upsilon$. We can choose such a system of coordinates where ${ }^{v} \Upsilon=\frac{1}{4}\left(e^{-\phi}\right)^{\diamond}$ and $h_{4}=\Phi$, i.e. this coefficient of the d-metric is considered as a generating function and the last conditions are solved.

A modification of the scale factor $\stackrel{\circ}{a}(t) \rightarrow a\left(x^{k}, t\right)$, for the FLRW metric (17) (with for $\stackrel{\circ}{g}_{1}=\stackrel{\circ}{g}_{2}=\stackrel{\circ}{g}_{3}=\stackrel{\circ}{a}^{2}, \stackrel{\circ}{g}_{4}=-1$, has to be chosen in order to explain observational cosmological data. For any prescribed functions $a\left(x^{k}, t\right)$ and $\omega^{2}=$ $\left|h_{4}\right|^{-1}$ and solutions $e^{\psi\left(x^{k}\right)}$, (see (24)) and $h_{a}[\widehat{\Phi}], n_{k}\left(x^{i}\right), w_{i}[\widehat{\Phi}]$ (given respectively by formulas (34)-(37)), we can compute the polarization functions $\eta_{i}=a^{-2} e^{\psi}, \eta_{3}=$ $\stackrel{\circ}{a}^{-2} h_{3}, \eta_{4}=1$ and function $\widehat{h}_{3}=h_{3} / a^{2}\left|h_{4}\right|$. Such coefficients (see the data (19)), define off-diagonal metrics of type (20),

$$
\begin{aligned}
d s^{2}= & a^{2}\left(x^{k}, t\right)\left[\eta_{1}\left(x^{k}, t\right)\left(d x^{1}\right)^{2}+\eta_{2}\left(x^{k}, t\right)\left(d x^{2}\right)^{2}\right] \\
& +a^{2}\left(x^{k}, t\right) \widehat{h}_{3}\left(x^{k}, t\right)\left[d y^{3}+n_{i}\left(x^{k}\right) d x^{i}\right]^{2}-\left[d t+\frac{\partial_{i} \Phi\left[\widehat{\Phi},{ }^{v} \Upsilon\right]}{\Phi^{\diamond}\left[\widehat{\Phi},{ }^{v} \Upsilon\right]} d x^{i}\right]^{2} .
\end{aligned}
$$

Choosing any generating functions $a^{2}\left(x^{k}, t\right), \psi\left(x^{i}\right)$ and $\Phi\left[\widehat{\Phi},{ }^{v} \Upsilon\right]$ and integration functions $n_{i}\left(x^{k}\right)$, we generate a nonhomogeneous cosmological model with nonholonomically induced torsion (14). More general torsions can be induced if $n_{i}\left(x^{k}, t\right)$ is taken with two types of integration functions ${ }_{1} n_{i}\left(x^{k}\right)$ and ${ }_{2} n_{i}\left(x^{k}\right)$ (see Eq. (36)). Having constructed this solution, we can now consider certain subclasses of generating and integration functions where $a\left(x^{k}, t\right) \rightarrow a(t) \neq \stackrel{\circ}{a}(t), w_{i} \rightarrow w_{i}(t), n_{i} \rightarrow$ const, etc. In this way generic off-diagonal cosmological metrics are generated (because there are nontrivial anholonomy coefficients $W_{i a}^{b}$. 


\subsubsection{Levi-Civita off-diagonal cosmological configurations}

The LC-conditions (32) are given by a set of nonholonomic constraints which cannot be solved in explicit form for arbitrary data $(\Phi, \Upsilon)$ and arbitrary integration functions ${ }_{1} n_{k}$ and ${ }_{2} n_{k}$. However, some subclasses of off-diagonal solutions can still be constructed where via frame and coordinate transforms we can chose ${ }_{2} n_{k}=0$ and ${ }_{1} n_{k}=\partial_{k} n$ with a function $n=n\left(x^{k}\right)$. It should be noted that $\left(\partial_{i}-w_{i} \partial_{4}\right) \Phi \equiv 0$ for any $\Phi\left(x^{k}, y^{4}\right)$ if $w_{i}$ is defined by (37). Introducing a new functional $B(\Phi)$, we find that $\left(\partial_{i}-w_{i} \partial_{4}\right) B=$ $\frac{\partial B}{\partial \Phi}\left(\partial_{i}-w_{i} \partial_{4}\right) \Phi=0$. Using Eq. (34) for functionals of type $h_{3}=B(|\tilde{\Phi}(\Phi)|)$, we solve Eq. $\left(\partial_{i}-w_{i} \partial_{4}\right) h_{3}=0$, what is equivalent to the second system of equations in (32), because $\left(\partial_{i}-w_{i} \partial_{4}\right) \ln \sqrt{\left|h_{3}\right|} \sim\left(\partial_{i}-w_{i} \partial_{4}\right) h_{3}$.

We can use a subclass of generating functions $\Phi=\check{\Phi}$ for which $\left(\partial_{i} \check{\Phi}\right)^{\diamond}=\partial_{i} \check{\Phi}^{\diamond}$ and get for the left part of the second equation in (32), $\left(\partial_{i}-w_{i} \partial_{4}\right) \ln \sqrt{\left|h_{3}\right|}=0$. The first system of equations in (32) can be solved in explicit form if $w_{i}$ are determined by formulas (37), and $h_{3}[\tilde{\Phi}]$ and $h_{4}\left[\tilde{\Phi}, \tilde{\Phi}^{\diamond}\right]$ are chosen respectively for any $\Upsilon \rightarrow \Lambda$. We can consider $\tilde{\Phi}=\tilde{\Phi}\left(\ln \sqrt{\left|h_{4}\right|}\right)$ for a functional dependence $h_{4}[\tilde{\Phi}[\check{\Phi}]]$. This allows us to obtain $w_{i}=\partial_{i}|\tilde{\Phi}| /|\tilde{\Phi}|^{\diamond}=\partial_{i}\left|\ln \sqrt{\left|h_{4}\right|}\right| /\left|\ln \sqrt{\left|h_{4}\right|}\right|^{\diamond}$. Taking the derivative $\partial_{4}$ on both sides of these equations, we get $w_{i}^{\diamond}=\frac{\left(\partial_{i} \mid \ln \sqrt{\left|h_{4}\right|}\right)^{\diamond}}{\left|\ln \sqrt{\left|h_{4}\right|}\right|^{\diamond}}-w_{i} \frac{\left|\ln \sqrt{\left|h_{4}\right|}\right|^{\diamond}}{\left|\ln \sqrt{\left|h_{4}\right|}\right|^{\diamond}}$. If the mentioned conditions are satisfied, we can construct in explicit form generic offdiagonal configurations with $w_{i}^{\diamond}=\left(\partial_{i}-w_{i} \partial_{4}\right) \ln \sqrt{\left|h_{4}\right|}$, which is necessary for the zero torsion conditions. We need also to solve for the conditions $\partial_{k} w_{i}=\partial_{i} w_{k}$ from the second line in (32). We find in explicit form the solutions for such coefficients,

$$
\check{w}_{i}=\partial_{i} \check{\Phi} / \check{\Phi}^{\diamond}=\partial_{i} \widetilde{A}
$$

with a nontrivial function $\widetilde{A}\left(x^{k}, y^{4}\right)$ depending functionally on the generating function $\check{\Phi}$.

Finally, we conclude that we generate LC-configurations for a class of off-diagonal cosmological metric type (10) for $\Upsilon=\breve{\Upsilon}=\Lambda, \Phi=\check{\Phi}=\tilde{\Phi}$ and ${ }_{2} n_{k}=0$ in (36) which are parameterized by quadratic elements

$$
\begin{aligned}
d s^{2}= & e^{\psi\left(x^{k}\right)}\left[\left(d x^{1}\right)^{2}+\left(d x^{2}\right)^{2}\right]+\frac{\check{\Phi}^{2}}{4|\Lambda|}\left[d y^{3}+\left(\partial_{k} n\left(x^{i}\right)\right) d x^{k}\right]^{2} \\
& -\frac{\left(\check{\Phi}^{\diamond}\right)^{2}}{|\Lambda| \check{\Phi}^{2}}\left[d t+\left(\partial_{i} \widetilde{A}[\check{\Phi}]\right) d x^{i}\right]^{2} .
\end{aligned}
$$

We can re-write such solutions in the form (38). This provides us a general procedure of off-diagonal deformations with $\stackrel{a}{a}(t) \rightarrow a\left(x^{k}, t\right)$ [see the FLRW metric (17)], resulting in nonhomogeneous cosmological metrics in GR. Prescribing a function $a\left(x^{k}, t\right)$, a generating function $\check{\Phi}\left(x^{k}, t\right)$ and a solution $e^{\psi\left(x^{k}\right)}$ (see (24)), we respectively compute the v-conformal factor and the polarization functions for $\widehat{h}_{3}=$ $h_{3} / a^{2}\left|h_{4}\right|=\breve{\Phi}^{4} / 4 a^{2}\left(\check{\Phi}^{\diamond}\right)^{2}, \omega^{2}=\left|h_{4}\right|^{-1}=|\Lambda| \breve{\Phi}^{2} /\left(\breve{\Phi}^{\diamond}\right)^{2}, \eta_{i}=a^{-2} e^{\psi}, \eta_{3}=$ $\stackrel{\circ}{a}^{-2} h_{3}=\check{\Phi}^{2} / 4|\Lambda| \stackrel{a}{ }^{2}, \quad \eta_{4}=1$. Such coefficients (see data (19)) transform the off- 
diagonal cosmological solutions (40) into metrics of type (20),

$$
\begin{aligned}
d s^{2}= & a^{2}\left(x^{k}, t\right)\left\{\left[\eta_{1}\left(x^{k}, t\right)\left(d x^{1}\right)^{2}+\eta_{2}\left(x^{k}, t\right)\left(d x^{2}\right)^{2}\right]+\widehat{h}_{3}\left(x^{k}, t\right)\left[d y^{3}\right.\right. \\
& \left.\left.+\left(\partial_{k} n\left(x^{i}\right)\right) d x^{k}\right]^{2}\right\}-\left[d t+\left(\partial_{i} \widetilde{A}[\check{\Phi}]\right) d x^{i}\right]^{2} .
\end{aligned}
$$

The dependence on the source $\Lambda$ is contained in explicit form, for instance, in the polarization $\eta_{3}$. This class of effective Einstein off-diagonal metrics $\mathbf{g}_{\alpha \beta}\left(x^{k}, t\right)$ define new nonhomogeneous cosmological solutions in GR as off-diagonal deformations of the FLRW cosmology. For certain well-defined conditions, one can find limits $\mathbf{g}_{\alpha \beta} \rightarrow \mathbf{g}_{\alpha \beta}\left(t, a(t), \widehat{h}_{3}(t), \breve{\Phi}(t), \eta_{i}(t)\right)$. This provides explicit geometric models of nonlinear off-diagonal anisotropic cosmological evolution which, with respect to Nadapted frames, describe $a(t)$ with modified re-scaling factors.

\subsection{Effective FLRW cosmology for $f$-modified gravity}

The anholonomic frame deformation method outlined in previous subsections can be applied for the generation of off-diagonal cosmological solutions of field equations of modified gravities, see (21). Redefining the generating functions via the transforms (33) and $\Phi \rightarrow \check{\Phi} \rightarrow \tilde{\Phi}$, we can generate off-diagonal cosmological configurations with $\widehat{\mathbf{R}}=4 \Lambda$, see (22) and (23). Such parameterizations of geometric data and sources are possible for certain general conditions via transforms of $\mathrm{N}$-adapted frames when the action functional functionally depends on $\Lambda$ and on the effective sources, $\widehat{\mathbf{f}}[\widehat{\mathbf{R}}(\Lambda), \widehat{\mathbf{T}}(\Lambda), \widehat{\mathbf{P}}]$, with $\widehat{\mathbf{P}}(t)=\widehat{\mathbf{R}}_{\alpha \beta} \widehat{\mathbf{T}}^{\alpha \beta}=-3 \stackrel{\circ}{ }\left(H^{2}+H^{\diamond}\right)$ and $H=a^{\diamond} / a$ with scaling factor $a(t)$ taken for some limits of a solution (38), or (41).

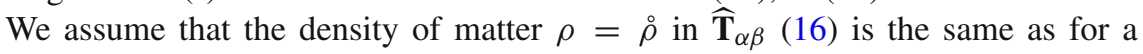
standard FLRW metric (17) and does not change under off-diagonal deformations with respect to $\mathrm{N}$-adapted frames. For such configurations, $\boldsymbol{\Theta}_{\beta}^{\alpha}=(p-2 \Lambda) \delta_{\beta}^{\alpha}$ and $\boldsymbol{\Xi}_{\beta}^{\alpha}=\left(2 \Lambda^{2}-p \Lambda-\frac{1}{2} 4 \Lambda^{2}\right) \delta_{\beta}^{\alpha}=-p \Lambda \delta_{\beta}^{\alpha}$, where terms with $\Lambda^{2}$ compensate each other in 4-d. We can write $\widehat{\mathbf{D}}_{\mu} \widehat{\mathbf{T}}_{\alpha \beta}=0, \widehat{\mathbf{D}}_{\mu}{ }^{1} \widehat{\mathbf{f}} \sim \partial^{2} \widehat{\mathbf{f}} / \partial \widehat{\mathbf{R}}_{\ldots}^{2} \mathbf{e}_{\mu} \Lambda \sim 0$, and (similarly) $\widehat{\mathbf{D}}_{\mu}{ }^{2} \widehat{\mathbf{f}} \sim 0, \widehat{\mathbf{D}}_{\mu}{ }^{3} \widehat{\mathbf{f}} \sim 0$, for $\widehat{\mathbf{R}}_{\alpha \beta} \sim \widehat{\mathbf{T}}_{\alpha \beta} \sim \Lambda \delta_{\alpha \beta}, \widehat{\Lambda}=$ const, with respect to corresponding classes of $\mathrm{N}$-adapted frames. Eq. (21) transform into a system of nonholonomic nonlinear PDEs of type (24)-(27), $\widehat{\mathbf{R}}_{\beta}^{\alpha}=\widehat{\boldsymbol{\Upsilon}} \delta_{\beta}^{\alpha}$, with effective diagonalized source

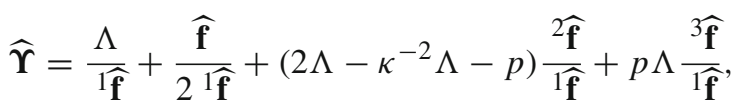

which can be parameterized with dependencies on $\left(x^{i}, t\right)$, or on $t$. These equations can be solved for very general off-diagonal forms, depending on generating and integration functions, following the procedure outlined in previous subsections. Redefining the generation function as in (33), when an effective cosmological constant $\check{\Lambda}$ is generated from $\widehat{\Upsilon}\left(x^{i}, t\right)$, one has $\check{\Phi}^{2}=\check{\Lambda}^{-1}\left[\widehat{\Phi}^{2}|\widehat{\Upsilon}|+\int d t \widehat{\Phi}^{2}|\widehat{\Upsilon}|^{\diamond}\right]$. Such a generating function defines off-diagonal cosmological solutions of type (38), or (40), as solutions of field equations for an effective (nonholonomic) Einstein space $\check{\mathbf{R}}_{\beta}^{\alpha}=\check{\Lambda} \delta^{\alpha}{ }_{\beta}$. In this 
way, a geometric method is provided when the (effective or modified) matter sources transform as $\widehat{\Upsilon}(22) \rightarrow \check{\Lambda}(23)$ and the gravitational field equations in modified gravity can be effectively expressed as nonholonomic Einstein spaces when the d-metric coefficients encode the contributions of $\widehat{\mathbf{f}},{ }^{1} \widehat{\mathbf{f}},{ }^{2} \widehat{\mathbf{f}}$ and ${ }^{3} \widehat{\mathbf{f}}$ and of the matter sources.

We can consider inverse transforms with $\breve{\Lambda} \rightarrow \widehat{\Upsilon}$ and state that for certain welldefined conditions (39) we can mimic both $f$-functional contributions and/or massive gravitational theories [41,42]. Here we emphasize that off-diagonal configurations (of vacuum and non-vacuum types) are possible even if the effective sources from modified gravity are constrained to be zero.

\section{Off-diagonal modeling of cosmological modified gravity theories}

This section has three goals. The first is to provide a reconstruction procedure for offdiagonal effective Einstein and modified gravity cosmological scenarios. The second is to apply these methods in practice and provide explicit examples related to $f(R)$ gravity and cosmology. The third goal is to analyze how matter stability problems for $f(R)$-theories can be solved by nonholonomic frame transforms and deformations and imposing non-integrable constraints.

\subsection{Reconstructing nonholonomic $f$-models}

Let us construct an effective Einstein space which models a quite general modified gravity theory with $f\left(R, T, R_{\alpha \beta} T^{\alpha \beta}\right)=R+F\left(R_{\alpha \beta} T^{\alpha \beta}\right)+G(T)$. This theory admits a reconstruction procedure which is similar to that elaborated in [20-22]. Following the anholonomic frame deformation method with an auxiliary canonical d-connection $\widehat{\mathbf{D}}$, the modified gravity (21) is formulated for

$$
\widehat{\mathbf{f}}\left(\widehat{\mathbf{R}}, \widehat{\mathbf{T}}, \widehat{\mathbf{R}}_{\alpha \beta} \widehat{\mathbf{T}}^{\alpha \beta}\right)=\widehat{\mathbf{R}}+\widehat{\mathbf{F}}(\widehat{\mathbf{P}})+\widehat{\mathbf{G}}(\widehat{\mathbf{T}}) .
$$

We can self-consistently embed this model into a nonholonomic background determined by $\mathrm{N}$-adapted frames (8) for a generic off-diagonal solution (41) with limits $\widehat{\mathbf{D}} \rightarrow \nabla$ and $\mathbf{g}_{\alpha \beta} \rightarrow \mathbf{g}_{\alpha \beta}\left(t, a(t), \widehat{h}_{3}(t), \breve{\Phi}(t), \eta_{i}(t)\right)$. With respect to such frames, the nonholonomic FLRW equations are similar to those found in section III B of [20-22] (see the second paper for details on methods of constructing solutions and speculations on the problem of matter instability). ${ }^{6}$

The effective function $a(t)$ defines in our case off-diagonal cosmological evolution scenarios which are different from those where $\stackrel{a}{a}(t)$ stands for a standard diagonal

\footnotetext{
6 In section III A of that work, a model with $G(T)=0$ was investigated in detail. The conclusion was that in order to elaborate a realistic evolution it is necessary to consider nontrivial values for $G(T)$. In nonholonomic variables, such term $\widehat{\mathbf{G}}(\widehat{\mathbf{T}})$ allows to encode $f(R)$ modified theories and related into certain off-diagonal configurations in GR, which simplifies the solution of the problem of matter instability (see Sect. 4.3).
} 
FLRW cosmology. For $H:=a^{\diamond} / a,{ }^{1} \widehat{\mathbf{G}}:=d \widehat{\mathbf{G}} / d \widehat{\mathbf{T}}$ and ${ }^{1} \widehat{\mathbf{F}}:=d \widehat{\mathbf{F}} / d \widehat{\mathbf{P}}$, we have

$$
\begin{aligned}
& 3 H^{2}+\frac{1}{2}\left[\widehat{\mathbf{f}}+\widehat{\mathbf{G}}-3\left(3 H^{2}-H^{\diamond}\right) \rho^{1} \widehat{\mathbf{F}}\right]-\rho\left(\kappa^{2}-{ }^{1} \widehat{\mathbf{G}}\right)=0, \\
& -3 H^{2}-2 H^{\diamond}-\frac{1}{2}\left[\widehat{\mathbf{f}}+\widehat{\mathbf{G}}-\left(\rho^{1} \widehat{\mathbf{F}}\right)^{\diamond \diamond}-4 H\left(\rho^{1} \widehat{\mathbf{F}}\right)^{\diamond}-\left(3 H^{2}+H^{\diamond}\right) \rho^{1} \widehat{\mathbf{F}}\right]=0 .
\end{aligned}
$$

An observer is here in a nonholonomic basis determined by $N_{i}^{a}=\left\{n_{i}, w_{i}(t)\right\}$ for a nontrivial off-diagonal vacuum with effective polarizations $\eta_{\alpha}(t)$, and can test cosmological scenarios in terms of the redshift $1+z=a^{-1}(t)$ for $P=P(z)$ and $T=T(z)$, with a new "shift" derivative when (for instance, for a function $s(t)) s^{\diamond}=-(1+z) H \partial_{z}$.

The system of two Eq. (44) simplifies by extending it to a set of three equations for four unknown functions $\{\widehat{\mathbf{f}}(z), \widehat{\mathbf{G}}(z), \rho(z), \varsigma(z)\}$ with a new variable $\varsigma(z):=\rho^{1} \widehat{\mathbf{F}}$,

$$
\begin{aligned}
& \left.3 H^{2}+\frac{1}{2} \widehat{\mathbf{f}}(z)+\widehat{\mathbf{G}}(z)\right]-\frac{3}{2}\left[3 H^{2}-(1+z) H\left(\partial_{z} H\right)\right] \varsigma(z) \frac{3}{2} H^{2}(1+z) \partial_{z} \varsigma(z) \\
& \quad-\kappa^{2} \rho(z)=0, \\
& -3 H^{2}+(1+z) H\left(\partial_{z} H\right)-\frac{1}{2} \widehat{\{}(z)+\widehat{\mathbf{G}}(z)-\left[3 H^{2}-(1+z) H\left(\partial_{z} H\right)\right] \varsigma(z) \\
& \left.+\left[3(1+z) H^{2}-(1+z) H\left(\partial_{z} H\right)\right] \partial_{z} \varsigma(z)+(1+z)^{2} \partial_{z z}^{2} \varsigma(z)\right\}=0, \\
& \left(\partial_{z}{ }^{1} \widehat{\mathbf{F}}\right) \varsigma(z)-\rho(z)\left(\partial_{z} \widehat{\mathbf{f}}\right)=0 .
\end{aligned}
$$

Here, by re-scaling the generating function, we have fixed the condition $\partial_{z}{ }^{1} \widehat{\mathbf{G}}(z)=0$. Such a nontrivial term must be considered if one wants to transform $\widehat{\mathbf{f}}$ into a standard theory $f\left(R, T, R_{\alpha \beta} T^{\alpha \beta}\right)$. The functional $\widehat{\mathbf{G}}(\widehat{\mathbf{T}})$, in both holonomic and nonholonomic forms, encodes a new degree of freedom for the evolution of the energy-density of type $\rho=\rho_{0} a^{-3(1+\varpi)}=\rho_{0}(1+z) a^{3(1+\varpi)}$, which is taken for the dust matter approximation $\varpi$ when the evolution reduces to $\rho \sim(1+z)^{3}$. For the assumption that such an evolution can be considered with respect to $\mathrm{N}$-adapted frames, the solutions of (45) are determined by data $\{\widehat{\mathbf{f}}(z), \widehat{\mathbf{G}}(z), \varsigma(z)\}$ by replacing the second and third equations into the first one and obtaining a single fourth-order equation for $\widehat{\mathbf{f}}(z)$.

The reconstruction procedure is restricted to fluids without pressure when such approximation is considered locally with $\mathrm{N}$-adapted frames and the expressions (18) for $(\stackrel{\circ}{a}, \stackrel{\circ}{H}, \stackrel{\rho}{\rho})$ are re-defined in terms of $(a, H, \rho)$; data are written with a script " 0 " if $z=z_{0}$, with $\xi=\kappa^{2} \rho_{0} / 3 H_{0}^{2}$. One should not confuse, e.g., $\stackrel{\circ}{H}$ and $H_{0}$, because these values are computed for different FLRW solutions, with $\stackrel{\circ}{a}(z)$ determined for a diagonal configuration and $a(z)$ for an off-diagonal one, respectively. We can express $\widehat{\mathbf{T}}=\widehat{\mathbf{T}}_{\alpha}^{\alpha}=-\xi \frac{3 H_{0}^{2}}{\kappa^{2}}(1+z)^{3}$ and $\widehat{\mathbf{P}}=\widehat{\mathbf{R}}_{\alpha \beta} \widehat{\mathbf{T}}^{\alpha \beta}=-3 \xi \frac{3 H_{0}^{2}}{\kappa^{2}}(1+z)^{3}\left[H^{2}-(1+\right.$ z) $H\left(\partial_{z} H\right)$ ]. Following the approach outlined in Sect. IIIB of [20-22], we introduce the parameterizations

$$
\widehat{\mathbf{F}}(\widehat{\mathbf{P}})=H_{0}^{2} \check{\mathbf{F}}(\check{\mathbf{P}}) \text { and } \widehat{\mathbf{G}}(\widehat{\mathbf{T}})=H_{0}^{2} \check{\mathbf{G}}(\check{\mathbf{T}}),
$$

where $\mathbf{P} \iota \widehat{\mathbf{P}} / P_{0}$ and $\mathbf{T}^{\iota}=\widehat{\mathbf{T}} / T_{0}$, for $P_{0}=-9 H_{0}^{4} \xi / \kappa^{2}$ and $T_{0}=-3 H_{0}^{2} \xi / \kappa^{2}$. In correspondingly $\mathrm{N}$-adapted variables, the off-diagonal cosmological solutions can be 
associated with a class of de Sitter (dS) solutions with effective cosmological constant $\check{\Lambda}$, where $H(z)=\check{H}_{0}$ results in $\check{\mathbf{P}}=\check{\mathbf{T}}=(1+z)^{3}$. In these variables, the solutions of (45) can be written as

$$
\begin{aligned}
\check{\mathbf{F}} & =c_{1} \check{\mathbf{P}}^{b_{1}}+\check{\mathbf{P}}^{b_{2} / 3}\left[c_{2} \cos \left(\frac{b_{3}}{3} \ln \check{\mathbf{P}}\right)+c_{3} \sin \left(\frac{b_{3}}{3} \ln \check{\mathbf{P}}\right)\right]+c_{4}+3 \xi \check{\mathbf{P}} \\
\check{\mathbf{G}} & =\tilde{c}_{1} \check{\mathbf{T}}^{b_{1}}+\check{\mathbf{T}}^{b_{2} / 3}\left[\tilde{c}_{2} \cos \left(\frac{b_{3}}{3} \ln \check{\mathbf{T}}\right)+\tilde{c}_{3} \sin \left(\frac{b_{3}}{3} \ln \check{\mathbf{P}}\right)\right]+\tilde{c}_{4}-3 \xi \check{\mathbf{T}}
\end{aligned}
$$

being the constants $b_{1}=-1.327, b_{2}=3.414$ and $b_{3}=1.380$. The values $c_{1}, c_{2}, c_{3}$ and $c_{4}$ are integration constants, and the second set of constants $\tilde{c}_{1}, \tilde{c}_{2}, \tilde{c}_{3}$ and $\tilde{c}_{4}$ can be expressed via such integration constants, and $b_{1}, b_{2}$ and $b_{3}$. We omit explicit formulas because for general solutions they can be included in certain generating or integration functions for the modified gravity equations and ultimately related to real observation data for the associated cosmological models.

For off-diagonal configurations, the $\widehat{\mathbf{f}}\left(\widehat{\mathbf{R}}, \widehat{\mathbf{T}}, \widehat{\mathbf{R}}_{\alpha \beta} \widehat{\mathbf{T}}^{\alpha \beta}\right)$ gravity positively allows for dS solutions in presence of non-constant fluids, not only due to the term $\widehat{\mathbf{P}}=\widehat{\mathbf{R}}_{\alpha \beta} \widehat{\mathbf{T}}^{\alpha \beta}$ in (15), and respective gravitational field and cosmological equations. This is possible also because of the off-diagonal nonlinear gravitational interactions in the effective gravitational models. It should be emphasized that the reconstruction procedure elaborated in [20-22], see also references therein, can be extended to more general classes of modified gravity theories, to Finsler like theories and the ensuing cosmological models [41,42]. Introducing (47) and (46) into (43), we reconstruct a function $\widehat{\mathbf{f}}=\widehat{\mathbf{R}}+\widehat{\mathbf{F}}(\widehat{\mathbf{P}})+\widehat{\mathbf{G}}(\widehat{\mathbf{T}})$. As a result, we can associate an effective matter source $\widehat{\boldsymbol{\Upsilon}}$, which allows the definition of a corresponding generating function $\check{\Phi}$ (see also $\Phi$ and (33)). Finally, we can reconstruct an off-diagonal cosmological solution with nonholonomically induced torsion of type (38) or to model a similar cosmological metric for LC configurations (40) (equivalently, (41)).

\subsection{How $f$-gravity and cosmology are encoded in nonholonomic configurations?}

It is well known that any FLRW cosmology can be realized in a specific $f(R)$ gravity (see Ref. [47-50] and, for further generalizations, [20-22]). ${ }^{7}$ In this subsection we analyze two examples of reconstruction of $f(R)$-gravities where the "e-folding" variable $\zeta:=\ln a / a_{0}=-\ln (1+z)$ is used instead of the cosmological time $t$ and in related nonholonomic off-diagonal deformations. For such models, we consider $\widehat{\mathbf{f}}=\widehat{\mathbf{f}}(\widehat{\mathbf{R}})$ in (15), use $\widehat{\boldsymbol{\Upsilon}}\left(x^{i}, \zeta\right)=\Lambda /{ }^{1} \widehat{\mathbf{f}}+\widehat{\mathbf{f}} / 2{ }^{1} \widehat{\mathbf{f}}$ instead of (42), which can be parameterized with dependencies on $\left(x^{i}, \zeta\right)$ (in particular, only on $\zeta$ ), $\check{\Phi}^{2}=\check{\Lambda}^{-1}\left[\widehat{\Phi}^{2}|\widehat{\Upsilon}|+\int d \zeta \widehat{\Phi}^{2} \partial_{\zeta}|\widehat{\Upsilon}|\right]$, when $\partial_{\zeta}=\partial / \partial \zeta$ with $s^{\diamond}=H \partial_{\zeta} s$ for any function $s$. The matter energy density $\rho$ is taken as in (45).

We restrict ourselves to $\mathrm{N}$-adapted frames (8), determined by an off-diagonal cosmological solution of the (modified) gravitational field equations, and can repeat all

\footnotetext{
7 We use a system of notations different from that article; here, e.g., $N$ in used for the N-connection and we work with nonholonomic geometric objects.
} 
computations leading to Eqs. (2)-(7) in [47-50] and prove that a modified gravity with $\widehat{\mathbf{f}}(\widehat{\mathbf{R}})$ realizes the FLRW cosmological model. Such solutions depend on the above source type $\widehat{\Upsilon}\left(x^{i}, \zeta\right)$ and generating function $\breve{\Phi}\left(x^{i}, \zeta\right)$; also the nonholonomic background can be modeled to be nonhomogeneous (via $w_{i}$ and $n_{i}$ depending respectively on $x^{i}$ and $\zeta$, or only on $\zeta$ ). The off-diagonal analog of the field equation corresponding to the first FLRW equation is

$\left.\left.\widehat{\mathbf{f}}(\widehat{\mathbf{R}})=\left(H^{2}+H \partial_{\zeta} H\right) \partial_{\zeta} \widehat{\mathbf{f}}(\widehat{\mathbf{R}})\right]-36 H^{2}\left[4 H+\left(\partial_{\zeta} H\right)^{2}+H \partial_{\zeta \zeta}^{2} H\right] \partial_{\zeta \zeta}^{2} \widehat{\mathbf{f}}(\widehat{\mathbf{R}})\right]+\kappa^{2} \rho$.

In terms of an effective quadratic Hubble rate, $q(\zeta):=H^{2}(\zeta)$, and considering that $\zeta=\zeta(\widehat{\mathbf{R}})$ for certain parameterizations, this equation yields

$$
\begin{aligned}
\widehat{\mathbf{f}}(\widehat{\mathbf{R}})= & -18 q(\zeta(\widehat{\mathbf{R}}))\left[\partial_{\zeta \zeta}^{2} q(\zeta(\widehat{\mathbf{R}}))+4 \partial_{\zeta} q(\zeta(\widehat{\mathbf{R}}))\right] \frac{d^{2} \widehat{\mathbf{f}}(\widehat{\mathbf{R}})}{d \widehat{\mathbf{R}}^{2}} \\
& +6\left[q(\zeta(\widehat{\mathbf{R}}))+\frac{1}{2} \partial_{\zeta} q(\zeta(\widehat{\mathbf{R}}))\right] \frac{d \widehat{\mathbf{f}}(\widehat{\mathbf{R}})}{d \widehat{\mathbf{R}}}+2 \rho_{0} a_{0}^{-3(1+\varpi)} a^{-3(1+\varpi) \zeta(\widehat{\mathbf{R}})}
\end{aligned}
$$

We can construct an off-diagonal cosmological model with metrics of type (38) and nonholonomically induced torsion (when $t \rightarrow \zeta$ ) if a solution $\widehat{\mathbf{f}}(\widehat{\mathbf{R}})$ is used for computing $\widehat{\Upsilon}$ and $\breve{\Phi}$. Modeling such nonlinear systems we can consider solutions of the field equations for an effective (nonholonomic) Einstein space $\check{\mathbf{R}}_{\beta}^{\alpha}=\check{\Lambda}^{\alpha}{ }_{\beta}^{\alpha}$, when certain terms of type $d \widehat{\mathbf{f}}(\check{\mathbf{R}}) / d \check{\mathbf{R}}$ and higher derivatives vanish for a functional dependence $\widehat{\mathbf{f}}(\breve{\Lambda})$ with $\partial_{\zeta} \breve{\Lambda}=0$. The nonholonomic cosmological evolution is determined by off-diagonal coefficients of the metrics and by certain non-explicit relations for the functionals variables, like $q(\zeta(\widehat{\mathbf{R}}(\breve{\Lambda})))$ and (effective/modified) matter sources transform as $\widehat{\Upsilon}(22) \rightarrow \breve{\Lambda}(23)$.

LC-configurations can be modeled by off-diagonal cosmological metrics of type (40) when the zero torsion conditions (32) are satisfied. We obtain a standard expression (see [47-50]) for the curvature of $\nabla$,

$$
R=3 \partial_{\zeta} q(\zeta)+12 q(\zeta)
$$

if the polarization or generating functions for (40) and the solutions of (48) are taken for diagonal configurations.

We here provide an example of reconstruction of models of $f(R)$ gravity and nonholonomically deformed GR when both reproduce the $\Lambda$ CDM era. For simplicity, we do not consider a real matter source (if such a source exists, it can be easily encoded into a nontrivial vacuum structure with generic off-diagonal contributions).

With respect to correspondingly $\mathrm{N}$-adapted frames and for $a(\zeta)$ and $H(\zeta)$ determined by an off-diagonal solution (38), with nonholonomically induced torsion, or (41), for LC-configurations, the FLRW equation for $\Lambda$ CDM cosmology is given by

$$
3 \kappa^{-2} H^{2}=3 \kappa^{-2} H_{0}^{2}+\rho_{0} a^{-3}=3 \kappa^{-2} H_{0}^{2}+\rho_{0} a_{0}^{-3} e^{-3 \zeta} .
$$


This equation looks similar to the one for Einstein gravity for diagonal configurations but contains values determined, in general, for other classes of models with off-diagonal interactions. Thus, $H_{0}$ and $\rho_{0}$ are fixed to be certain constant values, after the coefficients of off-diagonal solutions are found, and for an approximation were the dependencies on $\left(x^{i}, \zeta\right)$ are changed into dependencies on $\zeta$ (via a corresponding redefinition of the generating functions and the effective sources). We can relate the first term on the rhs to an effective cosmological constant $\Lambda$ (23), which in our approach appears via a re-definition (33). The second term in the formula describes, in general, an inhomogeneous distribution of cold dark mater (CDM) with respect to $\mathrm{N}$-adapted frames. In order to keep the similarity with the diagonalizable cosmological models in GR we can choose these integration constants for $\Lambda=12 H_{0}^{2}$ to survive in the limit $w_{i}, n_{i} \rightarrow 0$. It should be noted that such limit must be computed for "nonlinear" nonholonomic constraints via generating functions and effective sources.

Using (50), the effective quadratic Hubble rate and the modified scalar curvature, $\widehat{\mathbf{R}}$, are computed to be, respectively, $q(\zeta):=H_{0}^{2}+\kappa^{2} \rho_{0} a_{0}^{-3} e^{-3 \zeta}$ and $\widehat{\mathbf{R}}=3 \partial_{\zeta} q(\zeta)+$ $12 q(\zeta)=12 H_{0}^{2}+\kappa^{2} \rho_{0} a_{0}^{-3} e^{-3 \zeta}$. These functional formulas can be used for the dependencies on $\widehat{\mathbf{R}}$ if a necessary re-definition of the generation functions, or an approximation $\left(x^{i}, \zeta\right) \rightarrow \zeta$ is performed. Expressing $3 \zeta=-\ln \left[\kappa^{-2} \rho_{0}^{-1} a_{0}^{3}(\widehat{\mathbf{R}}-\right.$ $\left.\left.12 H_{0}^{2}\right)\right]$ and $X:=-3+\widehat{\mathbf{R}} / 3 H_{0}^{2}$, we obtain from Eq. (48)

$$
X(1-X) \frac{d^{2} \widehat{\mathbf{f}}}{d X^{2}}+\left[\chi_{3}-\left(\chi_{1}+\chi_{2}+1\right) X\right] \frac{d \widehat{\mathbf{f}}}{d X}-\chi_{1} \chi_{2} \widehat{\mathbf{f}}=0,
$$

for certain constants, for which $\chi_{1}+\chi_{2}=\chi_{1} \chi_{2}=-1 / 6$ and $\chi_{3}=-1 / 2$. The solutions of this equation with constant coefficients and for $R$ (49) were found in [47-50] as Gauss hypergeometric function, denoted there by $\widehat{\mathbf{f}}=F(X):=F\left(\chi_{1}, \chi_{2}, \chi_{3} ; X\right)$, as $F(X)=A F\left(\chi_{1}, \chi_{2}, \chi_{3} ; X\right)+B X^{1-\chi_{3}} F\left(\chi_{1}-\chi_{3}+1, \chi_{2}-\chi_{3}+1,2-\chi_{3} ; X\right)$ (for some constants $A$ and $B$ ). This provides a proof of the statement that $f(R)$ gravity can indeed describe $\Lambda$ CDM scenarios without the need of an effective cosmological constant. Working with auxiliary connections of the type $\widehat{\mathbf{D}}$, we can generalize the constructions to off-diagonal configurations and various classes of modified gravity theories, where $A, B$ and $\chi_{1}, \chi_{2}, \chi_{3}$ are appropriate functions of the $h$ coordinates. For instance, reconstruction procedures for Finsler like theories and cosmology models on tangent and Lorentz bundles, and bi-metric/massive gravity models are given in [39-42].

Having chosen $\widehat{\mathbf{f}}=F(X)$ for a modified gravity, we can go further and mimic an offdiagonal configuration when $\widehat{\mathbf{f}}=\widehat{\mathbf{f}}(\widehat{\mathbf{R}})$ is introduced in (15) and the source $\widehat{\Upsilon}\left(x^{i}, \zeta\right)=$ $\Lambda /{ }^{1} \widehat{\mathbf{f}}+\widehat{\mathbf{f}} / 2{ }^{1} \widehat{\mathbf{f}}$ is considered instead of (42) and (33) for $\check{\Phi}^{2}=\check{\Lambda}^{-1}\left[\widehat{\Phi}^{2}|\widehat{\mathbf{\Upsilon}}|+\right.$ $\left.\int d \zeta \widehat{\Phi}^{2} \partial_{\zeta}|\widehat{\Upsilon}|\right]$. Nevertheless, recovering nonhomogeneous modified cosmological models cannot be completed for general re-parameterized dependencies on $\left(x^{i}, \zeta\right)$ (in particular, only on $\zeta$ ). This distinguishes explicitly the modified gravity theories of type $f(R)$ from those generated by nonholonomic deformations. For certain homogeneity conditions, we can state an equivalence of some classes of gravities and cosmological models, or analyze their alternative physical implications. But a complete recovering is only possible if all generating and integration functions and the effective sources are correlated with certain observable cosmological effects and further approximations 
and re-definitions in terms of constant parameters and functionals depending on a time-like coordinate can be effectively performed.

The AFDM allows to reconstruct off-diagonal configurations modeling $f(R)$ gravity and cosmology with non-phantom or phantom matter in GR. With respect to $\mathrm{N}$-adapted frames in an off-diagonal (modified, or not) gravitational background, the FLRW equations can be written as

$$
3 \kappa^{-2} H^{2}={ }_{s} \rho\left(x^{k}\right) a^{-c\left(x^{k}\right)}+{ }_{p} \rho\left(x^{k}\right) a^{c\left(x^{k}\right)},
$$

where $a\left(x^{k}, \zeta\right)$ and $H\left(x^{k}, \zeta\right)$ are determined by a solution (38), or (41). For reparameterizations or approximations with $\left(x^{i}, \zeta\right) \rightarrow \zeta$, we assume that the positive functions ${ }_{s} \rho\left(x^{k}\right), p \rho\left(x^{k}\right)$ and $c\left(x^{k}\right)$ can be considered. The first term on the rhs dominates for small $a$ in the early universe, as in GR with non-phantom matter described by an EoS parameter $w=-1+c / 3>-1$. Introducing $q\left(x^{k}, \zeta\right):=H^{2}\left(x^{k}, \zeta\right)$ and the respective functionals ${ }_{s} q:=\frac{\kappa^{2}}{3}{ }_{s} \rho a_{0}^{-c}$ and ${ }_{p} q:=\frac{\kappa^{2}}{3}{ }_{p} \rho a_{0}^{c}$, for $q={ }_{s} q e^{-c \zeta}+$ ${ }_{p} q e^{c \zeta}$, in $\widehat{\mathbf{R}}=3 \partial_{\zeta} q(\zeta)+12 q(\zeta)$, we find

$$
e^{c \zeta}=\left\{\begin{array}{cc}
{\left[\widehat{\mathbf{R}} \pm \sqrt{\widehat{\mathbf{R}}^{2}-4\left(144-9 c^{2}\right)}\right] / 6(4+c),} & \text { for } c \neq 4 \\
\widehat{\mathbf{R}} / 24, & \text { for } c=4
\end{array}\right.
$$

The non-phantom matter may correspond to the case $c=4$ in (53), including radiation with $w=1 / 3$. Eq. (52) transform into a functional equation on $Y$ determined by changing the functional variable $\widehat{\mathbf{R}}^{2}=-576_{s} q_{p} q Y, 4 Y(1-Y) \frac{d^{2} \widehat{\mathbf{f}}}{d Y^{2}}+(3+Y) \frac{d \widehat{\mathbf{f}}}{d Y}-$ $2 \widehat{\mathbf{f}}=0$. This is again a functional variant (if we consider dependencies on $x^{k}$ ) of the generating Gauss' hypergeometric function, similarly to (51), which can be solved in explicit form.

For the case $c \neq 4$ in (53), we obtain models with phantom-like dominant components. A similar procedure as for deriving Eqs. (22) and (23) in [47-50], results in a functional generalization of the Euler equation, namely $\widehat{\mathbf{R}}^{2} \frac{d^{2} \widehat{\mathbf{f}}(\widehat{\mathbf{R}})}{d \widehat{\mathbf{R}}^{2}}+A \widehat{\mathbf{R}} \frac{d \widehat{\mathbf{f}}(\widehat{\mathbf{R}})}{d \widehat{\mathbf{R}}}+$ $B \widehat{\mathbf{f}}(\widehat{\mathbf{R}})=0$, for some coefficients $A=-H_{0}\left(1+H_{0}\right)$ and $B=\left(1+2 H_{0}\right) / 2$, for $H_{0}=1 / 3\left(1+{ }_{p h} w\right)$. Here we consider, for simplicity, homogenous limits and approximations $H^{2}(t)=\frac{\kappa^{2}}{3}{ }_{p h} \rho$ for the phantom EoS fluid-like states, ${ }_{p h} p={ }_{p h} w_{p h} \rho$, with ${ }_{p h} w<-1$. In both cases, with a trivial or a nontrivial nonholonomically induced torsion, there are solutions of the nonholonomic Euler equations above which can be expressed in the form $\widehat{\mathbf{f}}(\widehat{\mathbf{R}})=C_{+} \widehat{\mathbf{R}}^{m_{+}}+C_{-} \widehat{\mathbf{R}}^{m_{-}}$, for some integration constants $C_{ \pm}$and $2 m_{ \pm}=1-A \pm \sqrt{(1-A)^{2}-4 B}$. This reproduces with respect to $\mathrm{N}$-adapted frames the phantom dark energy cosmology with a behavior of the type $a(t)=a_{0}\left(t_{s}-t\right)^{-H_{0}}$, where $t_{s}$ is the so-called Rip time. If the generating functions for the off-diagonal cosmological solutions are chosen in a way such that the $\mathrm{N}$-connection coefficients $w_{i}$ and $n_{i}$ transform to zero, the solutions describe universes which end at a Big Rip singularity during $t_{s}$. Additionally to the former result that in the $f(R)$ theory no phantom fluid is needed, we conclude that for off-diagonal configurations we can effectively model such locally anisotropic cosmological configurations. 
One can encode and effectively model various types of cosmological solutions for modified gravity theories with $f(R)$ and/or $f\left(R, T, R_{\alpha \beta} T^{\alpha \beta}\right)$ functionals and their nonholonomic deformations. The cosmological reconstruction procedures can be elaborated for various types of viable modified gravity which may pass, or not, local gravitational tests and explain observational data for accelerating cosmology, dark energy and dark matter interactions [20-22,39-42,47-50]. Nevertheless, these theories exhibit certain specific problems as non-conservation of the energy-momentum tensors for the effective or physical matter fields.

In explicit form, we explain how the "non-conservation" problem can be solved for off-diagonal solutions with one Killing symmetry in the framework of $f(R, T)$ theories generalizing certain constructions from [51]. Following a similar procedure as in Sect. II of that work, but using the operator $\widehat{\mathbf{D}}$ instead of $\nabla$, for $\widehat{\mathbf{f}}=\widehat{\mathbf{f}}(\widehat{\mathbf{R}}, \widehat{\mathbf{T}})$, and considering an $\mathrm{N}$-adapted parametrization of the effective source $\widehat{\Upsilon}=$ const, we prove that

$$
\left(1+\frac{\kappa^{2}}{2 \widehat{\mathbf{f}}}\right) \widehat{\mathbf{D}}^{\alpha} \widehat{\mathbf{T}}_{\alpha \beta}=\frac{1}{2} \mathbf{g}_{\alpha \beta} \widehat{\mathbf{D}}^{\alpha} \widehat{\mathbf{T}}-\left(\widehat{\mathbf{T}}_{\alpha \beta}+\widehat{\Theta}_{\alpha \beta}\right) \widehat{\mathbf{D}}^{\alpha} \ln \left({ }^{2} \widehat{\mathbf{f}}\right)-\widehat{\mathbf{D}}^{\alpha} \widehat{\Theta}_{\alpha \beta}
$$

In these equations the values ${ }^{2} \widehat{\mathbf{f}}:=\partial \widehat{\mathbf{f}} / \partial \widehat{\mathbf{T}}$ and $\widehat{\Theta}_{\alpha \beta}=-2 \widehat{\mathbf{T}}_{\alpha \beta}-p \mathbf{g}_{\alpha \beta}$ are used, with an energy-momentum tensor (16) for nonholonomic flows of a perfect fluid. In general, $\widehat{\mathbf{D}}^{\alpha} \widehat{\mathbf{T}}_{\alpha \beta} \neq 0$ even for nonholonomic deformations of GR. Nevertheless, we can consider a subclass of off-diagonal configurations in $\widehat{\mathbf{f}}(\widehat{\mathbf{R}}, \widehat{\mathbf{T}})$ gravity when $\widehat{\Upsilon}$ $(22) \rightarrow \check{\Lambda}(23)$ and $\check{\Phi}^{2}=\check{\Lambda}^{-1}\left[\widehat{\Phi}^{2}|\widehat{\Upsilon}|+\int d \zeta \widehat{\Phi}^{2} \partial_{\zeta}|\widehat{\Upsilon}|\right]$ result in $\widehat{\mathbf{f}} \rightarrow \mathbf{f}=\mathbf{k}$ and effective $\check{\mathbf{R}}_{\beta}^{\alpha}=\check{\Lambda}^{\alpha}{ }_{\beta}$ which admit LC-solutions with zero torsion. For such nonholonomic distributions with $\widehat{\mathbf{D}} \rightarrow \nabla, \widehat{\mathbf{D}}^{\alpha} \widehat{\mathbf{T}}_{\alpha \beta} \rightarrow \check{\nabla} \check{\Lambda}=0$ and all terms on the lhs of (54) vanish, because they are nonholonomically equivalent to functionals of the effective cosmological constant $\check{\Lambda}$. Such conditions are satisfied in correspondingly $\mathrm{N}$-adapted frames and for canonical d-connections. The equations (54) generalize to nonholonomic forms the similar ones derived for the Levi-Civita connection $\nabla$ (see Eq. (10) in Ref. [51]).

\subsection{Nonholonomic constraints and matter instability}

There is another serious problem in modified gravities which is related to possible matter instabilities related to modifications of the gravitational actions. Even tiny modifications of GR may make the new model to posses unstable interior solutions (see, e.g., [52,53]). It was demonstrated however that there are viable $f(R)$ theories (with appropriated choices of the functional) where such instabilities may not occur [1-10,54-56]. The issue of instability and stabilization via additional nonholonomic constraints will be studied in our further works. In this section, we speculate how the AFDM can be applied to stability analysis in more general $f\left(R, T, R_{\alpha \beta} T^{\alpha \beta}\right)$ theories. The corresponding field equations are very difficult to solve even in a linear approximation [20-22], if we work in coordinate frames and with general functionals. In the nonholonomic variable formalism, the gravitational field equations in modified gravity theories posses the decoupling property exhibited above, which allows to 
encode $f(R, \ldots)$-modifications into off-diagonal nonholonomic configurations for the effective Einstein manifolds.

For a stability analysis, the trace equations where (21) are multiplied by $\mathbf{g}^{\mu \nu}$ are to be considered, namely

$$
-2 \widehat{\mathbf{f}}+\left(\widehat{\mathbf{R}}+3 \widehat{\mathbf{D}}^{\mu} \widehat{\mathbf{D}}_{\mu}\right)^{1} \widehat{\mathbf{f}}+(\widehat{\mathbf{T}}+\boldsymbol{\Theta})^{2} \widehat{\mathbf{f}}+\left(\frac{1}{2} \widehat{\mathbf{D}}^{\mu} \widehat{\mathbf{D}}_{\mu} \widehat{\mathbf{T}}+\widehat{\mathbf{D}}_{\mu} \widehat{\mathbf{D}}_{\nu} \widehat{\mathbf{T}}^{\mu \nu}+\mathbf{\Xi}\right)^{3 \widehat{\mathbf{f}}}=\kappa^{2} \widehat{\mathbf{T}},
$$

where ${ }^{1} \widehat{\mathbf{f}}:=\partial \widehat{\mathbf{f}} / \partial \widehat{\mathbf{R}},{ }^{2} \widehat{\mathbf{f}}:=\partial \widehat{\mathbf{f}} / \partial \widehat{\mathbf{T}}$ and ${ }^{3} \widehat{\mathbf{f}}:=\partial \widehat{\mathbf{f}} / \partial \widehat{\mathbf{P}}$, when $\widehat{\mathbf{P}}=\widehat{\mathbf{R}}_{\alpha \beta} \widehat{\mathbf{T}}^{\alpha \beta}$. Let us envisage a trace configuration in the interior of a celestial body, when $\widehat{\mathbf{T}}=\widehat{\mathbf{T}}_{0}$ and $-2 \widehat{\mathbf{f}}+\widehat{\mathbf{R}}_{0}\left({ }^{1} \widehat{\mathbf{f}}\right)=\kappa^{2} \widehat{\mathbf{T}}_{0}$. Imposing nonholonomic constraints, we parameterize a LC-configuration in GR and model an interior solution in the presence of some gravitational objects (for instance, the Sun or the Earth). The $f$-modifications (in general with strong coupling for the curvature and the energy-momentum tensor) may result in a worsening of the stability problems and may prevent $\widehat{\mathbf{T}}_{0}$ to be a solution of any suitable background equation. It is difficult to find solutions of (55) even for very much simplified cases in the nonlinear situation if we work in coordinate frames for the connection $\widehat{\mathbf{D}}=\nabla$.

A rigorous study of the problem of matter instability for $f(R)$ and more generally $f\left(R, T, R_{\alpha \beta} T^{\alpha \beta}\right)$ gravities, for certain illustrative cases when $1 \widehat{\mathbf{f}}=R$, and for restrictive conditions where there is a qualitative description via additional functionals on $T$ and $P$ shows that the appearance of a large instability can actually be avoided. Using the AFDM, we can consider modified gravity theories with $f$ modifications which are effectively described by $\check{\mathbf{R}}_{\beta}^{\alpha}=\check{\Lambda}^{\alpha}{ }_{\beta}^{\alpha}$ when the modifications are encoded into polarization functions and $\mathrm{N}$-coefficients. For models generated by $\widehat{\mathbf{f}}\left(\widehat{\mathbf{R}}, \widehat{\mathbf{T}}, \widehat{\mathbf{R}}_{\alpha \beta} \widehat{\mathbf{T}}^{\alpha \beta}\right)=\widehat{\mathbf{f}}_{1}(\widehat{\mathbf{R}})+\widehat{\mathbf{F}}(\widehat{\mathbf{P}})+\widehat{\mathbf{G}}(\widehat{\mathbf{T}})$, we take a constant interior solution with $\widehat{\mathbf{T}}_{0}=$ const and $\widehat{\mathbf{P}}_{0}=$ const, and denote by $\widehat{\mathbf{f}}_{1}^{(s)}:=\partial^{s} \widehat{\mathbf{f}}_{1} / \partial \widehat{\mathbf{R}}^{s}$ and $\widehat{\mathbf{F}}^{(s)}:=\partial^{s} \widehat{\mathbf{F}} / \partial \widehat{\mathbf{P}}^{s}$ for $s=1,2, \ldots$ We can repeat, with respect to the $\mathrm{N}$-frames (8), the computations presented in detail for Eqs. (45)-(48) in [20-22] (see also references therein), and prove that Eqs. (55) for linear pertubations can be written in the form

$$
\begin{aligned}
& {\left[\check{\mathbf{D}}^{\mu} \check{\mathbf{D}}_{\mu}+2 \frac{\check{\mathbf{T}}_{0}^{\mu v}}{\check{\mathbf{T}}_{0}} \check{\mathbf{D}}_{\mu} \check{\mathbf{D}}_{v}+2 \frac{\Xi_{0}}{\check{\mathbf{T}}_{0}}+4 \frac{\check{\mathbf{P}}_{0}}{\check{\mathbf{T}}_{0}} \frac{\widehat{\mathbf{F}}_{1}^{(1)}}{\widehat{\mathbf{F}}^{(2)}}\right] \delta \check{\mathbf{P}}} \\
& =\left[\frac{2}{\check{\mathbf{T}}_{0}} \frac{\widehat{\mathbf{F}}^{(1)}}{\widehat{\mathbf{F}}^{(2)}}-\frac{\check{\mathbf{P}}_{0}}{\check{\mathbf{T}}_{0}} \widehat{\mathbf{F}}^{(1)} \widehat{\mathbf{F}}^{(2)}\left(2^{m} \widehat{\mathcal{L}}-\check{\mathbf{T}}_{0}\right)\right] \delta \check{\mathbf{R}} .
\end{aligned}
$$

The values of type $\delta \widehat{\mathbf{P}}$ and $\delta \widehat{\mathbf{R}}$ are considered for a small perturbations in the curvature where $\widehat{\mathbf{R}}=\widehat{\mathbf{R}}_{0}+\delta \widehat{\mathbf{R}}$ and $\widehat{\mathbf{P}}=\widehat{\mathbf{P}}_{0}+\delta \widehat{\mathbf{P}}$. No instability appears if $\delta \mathbf{P}=\delta \check{\mathbf{R}}=0$ which is a particular solution of the above equation. We can in fact model a damped oscillator with additional nonholonomic constraints if $\boldsymbol{\Xi}_{0}+2 \check{\mathbf{P}}_{0} \widehat{\mathbf{f}}_{1}^{(1)} / \widehat{\mathbf{F}}^{(2)} \geq \check{\mathbf{T}}_{0}$, which allows to avoid large instabilities in the interior of a spherical body. For some specific functionals $f(R)$ and appropriate $G(T)$, the same behavior as in GR results (with mass stability in the cosmological context), although there are possible large perturbations $\delta R$ and $\delta P$ remaining. The ideas how to circumvent the mass instability problem for holonomic 
configurations has been studied in [57-62]. Redefining the generating functions and sources in a $f$-modified model into an effective Einsteinian theory, with $\mathcal{S}[\check{\mathbf{R}}, \check{\Lambda}]$, one can consider a nonholonomically deformed Hilbert-Einstein action with $\widehat{\mathbf{f}} \rightarrow \mathbf{f}=\mathbf{L}_{R}$ In such cases, $\delta \widehat{\mathbf{R}}=\delta \mathbf{R}=0$ and instabilities are not produced, indeed, if we impose the zero torsion conditions (see (14)), we get back to the GR theory. Even if Eq. (55) involves not only perturbations of the Ricci scalar $\widehat{\mathbf{R}}$ but also of the Ricci d-tensor $\widehat{\mathbf{R}}_{\alpha \beta}$ (through $\delta \widehat{\mathbf{P}}$ ), via nonholonomic transforms to effective $\check{\mathbf{R}}_{\beta}^{\alpha}=\check{\Lambda}_{\beta}^{\alpha}{ }_{\beta}$, the stability of the system is obtained via off-diagonal interactions and the nonholonomic constraints used for an effective modeling of a subclass of $\widehat{\mathbf{f}}$-theories to certain nonholonomic deformations of the Einstein equations with effective cosmological constant $\breve{\Lambda}$. This is indeed a remarkable result.

\section{Concluding remarks and discussion}

We have presented a study of the conditions when a wide class of $f(R, \ldots)$ modified gravity theories, MGTs, can be encoded into effective off-diagonal Einstein spaces if nonholonomic deformations and constraints are considered for the nonlinear dynamics of gravity and matter fields. A special attention has been paid to a new version of MGT which includes strong coupling of the fields [20-22]. Notably, we advocated such theories to have physical motivations from the covariant Hořava-Lifshitz like gravity models, with dynamical breaking of the Lorentz invariance [11-14]. This provides also an example of a covariant, power-counting renormalizable theory and is represented by a simplest power-law $f$-gravity.

It is worthwhile to mention that the gravitational field equations in such MGTs admit a decoupling property with respect to certain classes of nonholonomic frames, which allows us to generate exact solutions for very general off-diagonal forms. The corresponding integral varieties of solutions are parameterized by generating and integration functions and various classes of commutative and noncommutative symmetry parameters. It is possible to re-define the generating functions and effective sources of matter fields in such a way that the $f$-terms are equivalently encoded into effective Einstein spaces with complex parametric nonlinear structure for the gravitational vacuum. We argue that certain nonholonomic configurations model also covariant gravity theories with nice ultraviolet behaviors and seem to be (super-)renormalizable in the sense of Hořava-Lifshitz gravity [11-19,41,42].

Notwithstanding the fact that the various $f(R)$ modified theories and general relativity, GR, are actually very different theories, the off-diagonal configurations and nonlinear parametric interactions considered in GR may encode various classes of such modified gravity effects and explain alternatively observational data for accelerating cosmology and certain effects in dark energy and dark matter physics. In both cases, it is possible to find cosmological solutions and reconstruct the corresponding action. In the already mentioned classes of modified gravity theories with $f$-modifications [1-14,20-22], the dynamics of the matter sector is modeled by a perfect fluid.

We note, finally, that MGTs in general contain ghosts, due to the higher-derivative terms in the action. However, we can select certain ghost-free configurations determined by corresponding classes of nonholonomic deformations or constraints. Such 
models of bi-metric and massive graviton gravities were recently studied in [3942]. Together with the results in [11-19], the conclusion is reached that some $f\left(R, T, R_{\alpha \beta} T^{\alpha \beta}\right)$ models, and their off-diagonal nonholonomic equivalents, may possess nice ultraviolet properties and that interesting connections can be established with viable theories of quantum gravity.

Acknowledgments This work has been partially supported by the Program IDEI, PN-II-ID-PCE-2011-30256, by an associated visiting research position at CERN, by MINECO (Spain), Grants PR2011-0128 and FIS2013-44881-P, by the CPAN Consolider Ingenio Project, and by AGAUR (Generalitat de Catalunya), contract 2009SGR-994. We thank S. Capozziello, S. D. Odintsov, S. Rajpoot, E. Saridakis, D. Singleton, and P. Stavrinos for important discussions and support.

\section{References}

1. Capozziello, S., Faraoni, V.: Beyond Einstein Gravity: A Survey of Gravitational Theories for Cosmology and Astrophysics, Fundamental Theories of Physics, vol. 170, p. 467. Springer, Netherlands (2011)

2. Capozziello, S., De Laurentis, M.: Phys. Rep. 509, 167 (2011)

3. Nojiri, S., Odintsov, S.D.: Phys. Rep. 505, 59 (2011)

4. Elizalde, E., Nojiri, S., Odintsov, S.D.: Phys. Rev. D 70, 043539 (2004)

5. Cognola, G., Elizalde, E., Odintsov, S.D., Tretyakov, P., Zerbini, S.: Phys. Rev. D 79, 044001 (2009)

6. Brevik, I., Elizalde, E., Nojiri, S., Odintsov, S.D.: Phys. Rev. D 84, 103508 (2011)

7. Crofton, T., Ferreira, P.G., Padilla, A., Skordis, C.: Phys. Rep. 513, 1 (2012)

8. Vitagliano, V., Sotiriou, T.P., Liberati, S.: Phys. Rev. D 82, 084007 (2010)

9. Sotiriou, T.P., Faraoni, V.: Rev. Mod. Phys. 82, 451-497 (2010)

10. Lobo, F.S.N., Martinez-Asencio, J., Olmo, G.J., Rubiera-Garcia, D.: Phys. Rev. D 90, 024033 (2014)

11. Nojiri, S., Odintsov, S.D.: Phys. Rev. D 81, 043001 (2010)

12. Nojiri, S., Odintsov, S.D.: Phys. Rev. D 83, 023001 (2011)

13. Kluson, J., Nojiri, S., Odintsov, S.D.: Phys. Lett. B 701, 117 (2011)

14. Elizalde, E., Nojiri, S., Odintsov, S.D., Saez-Gomez, D.: Eur. Phys. J. C 70, 351 (2010)

15. Vacaru, S.: EPL 96, 50001 (2011)

16. Vacaru, S.: Gen. Relativ. Gravit. 44, 1015 (2012)

17. Vacaru, S.: J. Math. Phys. 54, 073511 (2013)

18. Vacaru, S.: J. Geom. Phys. 60, 1289 (2010)

19. Vacaru, S.: Int. J. Geom. Meth. Mod. Phys. 6, 873 (2009)

20. Harko, T., Lobo, F.S.N., Nojiri, S., Odintsov, S.D.: Phys. Rev. D 84, 024020 (2011)

21. Odintsov, S.D., Saez-Gomez, D.: Phys. Lett. B 725, 437 (2013)

22. Haghani, Z., Harko, T., Lobo, F.S.N., Sepangi, H.R., Shahidi, S.: Phys. Rev. D 88, 044023 (2013)

23. Gheorghiu, T., Vacaru, O., Vacaru, S.: Eur. Phys. J. C 74, 3152 (2014)

24. Vacaru, S., Veliev, E., Yazici, E.: Int. J. Geom. Meth. Mod. Phys. 11, 1450088 (2014)

25. Vacaru, S., Veliev, E., Yazici, E.: J. Math. Phys. 46, 042503 (2005)

26. Vacaru, S., Veliev, E., Yazici, E.: J. Math. Phys. 49, 043504 (2008)

27. Vacaru, S., Veliev, E., Yazici, E.: JHEP 04, 009 (2001)

28. Vacaru, S., Singleton, D.: Class. Quant. Grav. 19, 3583 (2002)

29. Vacaru, S., Popa, F.C.: Class. Quant. Grav. 18, 4921 (2001)

30. Vacaru, S.: Int. J. Mod. Phys. D 21, 1250072 (2012)

31. Bailakos, S., Mavromatos, N.E., Mitsou, V.A., Plionis, M.: Astropart. Phys. 36, 7 (2012)

32. Kouretsis, A.P., Stathakopoulos, M., Stavrinos, P.C.: Phys. Rev. D 86, 124025 (2012)

33. Hohmann, M.: Phys. Rev. D 87, 124034 (2013)

34. Castro, C.: Int. J. Theor. Phys. 51, 3318 (2012)

35. Kostelecky, V.A., Russell, N., Tso, R.: Phys. Lett. B716 470 (2012)

36. Lammerzahl, C., Perlick, V., Hasse, W.: Phys. Rev. D 86, 104042 (2012)

37. Kramer, D., Stephani, H., Herdlt, E., MacCallum, M.A.H.: Exact Solutions of Einstein's Field Equations, 2nd edn. Cambridge University Press, Cambridge (2003) 
38. Griffiths, J.B., Podolský, J.: Exact Space-Times in Einstein's General Relativity. Cambridge University Press, Cambridge (2009)

39. Klusoň, J., Nojiri, S., Odintsov, S.D.: Phys. Lett. B 726, 918 (2013)

40. Vacaru, S.: Off-diagonal ekpyrotic scenarios and equivalence of modified, massive and/or Einstein gravity. arXiv: 1304.1080

41. Stavrinos, P., Vacaru, S.: Class. Quant. Grav. 30, 055012 (2013)

42. Vacaru, S.: Int. J. Geom. Meth. Mod. Phys. 11, 1450032 (2014)

43. Ellis, G.F.R.: Inhomogeneity effects in cosmology. arXiv: 1103.2335

44. Gödel, K.: Rev. Mod. Phys. 21, 447-450 (1949)

45. Krasiński, A.: Inholomogeneous Cosmological Models. Cambridge University Press, Cambridge (1997)

46. Appleby, S.A., Linder, E.V.: Phys. Rev. D 87, 023532 (2013)

47. Nojiri, S., Odintsov, S.D., Saez-Gomez, D.: Phys. Lett. B 681, 74 (2009)

48. Elizalde, E., Hurtado, J.Q.: Mod. Phys. Lett. A 19, 29 (2004)

49. Dunsby, P.K.S., Elizalde, E., Gowami, R., Odintov, S., Saez-Gomez, D.: Phys. Rev D 82, 023519 (2010)

50. de la Cruz-Dombriz, A., Dobado, A.: Phys. Rev. D 74, 087501 (2006)

51. Alvarenga, F.G., de la Cruz-Dombriz, A., Houndjo, M.J.S., Rodrigues, M.E., Saez-Gomez, D.: Phys. Rev. D 87, 103526 (2013)

52. Dolgov, A.D., Kawasaki, M.: Phys. Lett. B 573, 1 (2003)

53. Faraoni, V.: Phys. Rev. D 74, 104017 (2006)

54. Nojiri, S., Odintsov, S.D.: Phys. Rev. D 68, 123512 (2003)

55. Tamanini, N., Koivisto, T.S.: Phys. Rev. D 88, 064052 (2013)

56. Ayuso, I., Jimenez, J.B., de la Cruz-Dobriz, A.: arXiv: 1411.1636

57. Starobinsky, A.: JETP Lett. 86, 157 (2007)

58. Hu, W., Sawicki, I.: Phys. Rev. D 76, 064004 (2007)

59. Nojiri, S., Odintsov, S.D.: Phys. Rev. D 77, 026007 (2008)

60. Capozziello, S., Tsujikawa, S.: Phys. Rev. D 77, 107501 (2008)

61. Cognola, G., Elizalde, E., Nojiri, S., Odintsov, S.D., Sebastiani, L., Zerbini, S.: Phys. Rev. D 77, 046009 (2008)

62. Bamba, K., Geng, C.-Q., Lee, C.-C.: JCAP 1011, 001 (2010) 